1 Hacettepe Journal of Mathematics and Statistics

$\bigcap$ Volume 48 (3) (2019), 818-844

ResearCh ARTICLE

\title{
The power-linear hazard rate distribution and estimation of its parameters under progressively type-II censoring
}

\author{
Bahman Tarvirdizade*(i) and Nader Nematollahi ${ }^{\dagger \ddagger}$ (i)
}

\begin{abstract}
In this paper, we introduce a class of distributions which generalizes the power hazard rate distribution and is obtained by combining the linear and power hazard rate functions. This class of distributions, which is called the power-linear hazard rate distribution, is simple and flexible and contains some important lifetime distributions. The maximum likelihood estimators of the parameters using the NewtonRaphson (NR) and the expectation-maximization (EM) algorithms and the Bayes estimators of the parameters under squared error loss (SEL), linear-exponential (LINEX) and Stein loss functions are obtained based on progressively type-II censored sample. Also, we obtain the asymptotic confidence interval and some bootstrap confidence intervals and construct the HPD credible interval for the parameters. A real data set is analyzed and observed that the present hazard rate distribution can provide a better fit than other three-parameter distributions. Finally, a Monte Carlo simulation study is conducted to investigate and compare the performance of different types of estimators presented in this paper.
\end{abstract}

Keywords: Bayes estimation, Bootstrap confidence intervals, EM algorithm, MCMC methods, Power-linear hazard rate distribution, Progressive type-II censoring.

Mathematics Subject Classification (2010): 62E15, 62F10, 62F15

Received: 09.10.2017 Accepted: 28.06.2018 Doi: 10.15672/HJMS.2018.608

\footnotetext{
*Department of Statistics, Allameh Tabataba'i University, Tehran, Iran, Email: bahtary@gmail.com

${ }^{\dagger}$ Department of Statistics, Allameh Tabataba'i University, Tehran, Iran, Email: nematollahi@atu.ac.ir

$\ddagger$ Corresponding Author.
} 


\section{Introduction}

The hazard rate function plays an important role in reliability studies. The hazard rate measures the propensity to fail or to die depending on the age reached and it thus plays a key role in characterizing the process of aging and in classifying lifetime distributions. The hazard rate function describe the failure rate of the life test. It is known as the force of mortality or force of decrement in actuarial work and the intensity function in extreme value theory. The term age-specific death rate has also been used in vital statistics and in economics its reciprocal is known as Mill's rate. The hazard rate function is used in reliability applications to describe the instantaneous failure rate at any time (Rinne [27]). If the life time of a unit be a random variable $X$ with probability density function (pdf) $f(x)$ and cumulative distribution function (cdf) $F(x)$, then the hazard rate function $h(x)$ is defined by

$$
h(x)=\frac{f(x)}{1-F(x)} .
$$

In reliability theory, lifetime distributions are often specified by choosing a particular hazard rate function. One of these hazard rate functions is the linear hazard rate function which has been considered by several researchers. The linear hazard rate function has the form

$$
h(x)=a+b x, \quad x>0, \quad a, b \geq 0,
$$

where $a$ and $b$ are the parameters such that $a+b>0$. The linear hazard rate function induce the pdf

$$
f(x)=(a+b x) e^{-\left(a x+\frac{b}{2} x^{2}\right)}, \quad x>0, \quad a, b \geq 0,
$$

which is known as linear hazard rate distribution (LHRD). The linear hazard rate function was first introduced by Bain [2]. He provided a comprehensive statistical inference procedures for the estimation of the parameters $a$ and $b$ based on complete samples. The Bayes estimation of the parameters of LHRD is considered by Pandey et al. [23]. Sen and Bhattacharyya [30] studied the maximum likelihood and least squares type estimation based on type II censored samples from the LHRD, while Lin et al. [15] computed the maximum likelihood estimates of the parameters of the LHRD based on records and inter-record times. Monte Carlo methods for Bayesian inference on the LHRD are discussed by Lin et al. [16]. Bayesian inference of the LHRD under a progressively censoring scheme is also discussed by Sen et al. [31]. Recently, some authors considered generalizations of the LHRD for obtaining more flexible new families of distributions (see, e.g. Ghitany and Kotz [11], Sarhan and Kundu [28] and Cordeiro et al. [7]).

Another type of hazard rate function is the power hazard rate function which is defined as

$$
h(x)=a x^{k}, \quad x>0, \quad a>0, \quad k>-1,
$$

and induce the power hazard rate distribution (PHRD) with pdf

$$
f(x)=a x^{k} e^{-\frac{a}{k+1} x^{k+1}}, \quad x>0, \quad a>0, \quad k>-1 .
$$

Mugdadi [20] obtained the least squares type estimators of the parameters of the PHRD. Mugdadi and Min [21] investigated the Bayes estimation of the power hazard rate function. The problem of estimating the stress-strength reliability for the PHRD is considered by Kinaci [13].

Although the linear and power hazard rate distributions are very useful for modeling lifetime data, they have monotone hazard rates and are not reasonable to apply to phenomena with non-monotone hazard rates. In this paper, by combining the linear hazard rate function and the power hazard rate function, we consider a more general hazard 
rate function which can also cover non-monotone hazard rate. We define the power-linear hazard rate function in the form

$$
h(x)=a x^{k}+b x, \quad x>0, \quad a, b \geq 0, \quad k>-1,
$$

where $a, b$ and $k$ are the parameters such that $a+b>0$ and $k \neq 1$ (which gives the identifiability of the parameters of the new hazard rate function).

The power-linear hazard rate function is very simple and can cover constant, decreasing, increasing and bathtub-shaped hazard rates, while the hazard rate functions of most of the three-parameter distributions are either complicated (see, for example, the distributions discussed in Section 5) or cannot cover non-monotone cases (for example, the Gompertz-Makeham distribution by Makeham [18] and the Modified Weibull distribution by Sarhan and Zeindin [29]). We obtain the cdf and the pdf corresponding to this hazard rate function and call it the power-linear hazard rate distribution (P-LHRD). The maximum likelihood estimates (MLEs) of the unknown parameters via the NR and the EM algorithms and the Bayes estimators of the parameters under different loss functions based on progressively type-II censored data are derived. The asymptotic and bootstrap confidence intervals of the parameters and the highest posterior density (HPD) credible intervals are also obtained. A real data set is analyzed to illustrate application of the proposed distribution and to show that the present hazard rate distribution can provide a better fit than some three-parameter distributions. The different proposed methods have been compared by using a Monte Carlo simulation study.

The rest of the paper is organized as follows. In Section 2, we present the power-linear hazard rate distribution. In Section 3, the MLEs of the unknown parameters via the NR and the EM algorithms and the Bayes estimates of the parameters under SEL, LINEX and Stein loss functions based on progressively type-II censored sample are considered. In Section 4, we obtain the asymptotic and bootstrap confidence intervals and construct the HPD credible interval for the parameters of the P-LHRD. The application of the P-LHRD is illustrated by analyzing a real data set in Section 5. A simulation study is presented in Section 6. Finally, some conclusions are given in Section 7.

\section{The P-LHRD}

Suppose $X$ denotes the random variable having hazard rate function given in (1.1). Then the cdf and reliability function of $X$ are given by

$$
\begin{gathered}
F(x)=1-e^{-\left(\frac{b}{2} x^{2}+\frac{a}{k+1} x^{k+1}\right)}, \quad x>0, \quad a, b \geq 0, \quad k>-1, k \neq 1, \\
R(t)=1-F(t)=e^{-\left(\frac{b}{2} x^{2}+\frac{a}{k+1} x^{k+1}\right)},
\end{gathered}
$$

respectively, and the pdf of $X$ is given by

$$
f(x)=\left(b x+a x^{k}\right) e^{-\left(\frac{b}{2} x^{2}+\frac{a}{k+1} x^{k+1}\right)}, \quad x>0, \quad a, b \geq 0, \quad k>-1, k \neq 1 .
$$

For the random variable $X$ with pdf given by (2.2), we write $X \sim \operatorname{P-LHRD}(b, a, k)$. Taking the first derivative of the hazard rate function of the P-LHRD given in (1.1), we have $h^{\prime}(x)=b+a k x^{k-1}, x>0$. Hence, it can be easily verify that the hazard rate function of this distribution has a bathtub-shape when $a, b>0$ and $k<0$, and reaches a minimum at $x=(-b / a k)^{1 /(k-1)}$. The hazard rate function of this distribution can also be constant, increasing or decreasing for the other parameter values. Figure 1 shows different patterns of the probability density function and the hazard rate function of the P-LHRD for selected values of the parameters $b, a$ and $k$. 

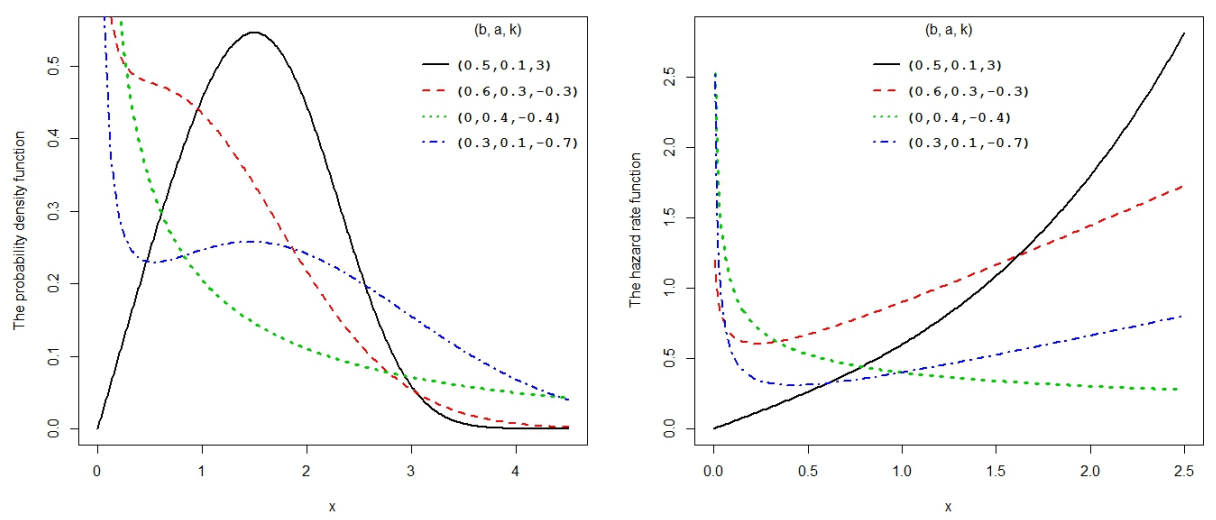

Figure 1. The probability density function and the hazard rate function of the P-LHRD for different parameter values.

The P-LHRD contains the following well-known lifetime distributions:

(1) When $b=k=0$, the P-LHRD reduces to the exponential distribution with parameter $a$.

(2) When $a=0$, the P-LHRD reduces to the Rayleigh distribution with parameter $1 / b$.

(3) When $k=0$, the P-LHRD reduces to the LHRD with parameters $a$ and $b$.

(4) When $k=2$, the P-LHRD reduces to the quadratic hazard rate distribution with parameters $0, a$ and $b$ introduced by Bain [2].

(5) When $b=0$, the P-LHRD reduces to the PHRD with parameters $a$ and $k$.

(6) When $b=0, k=\alpha-1$ and $a=\alpha / \theta$, the P-LHRD reduces to the Weibull distribution with parameters $\alpha$ and $\theta$.

The $r$ th moment $\left(\mu_{r}\right)$ of the $\operatorname{P-LHRD}(b, a, k)$ by using $(2.2)$ is given by

$$
\mu_{r}=E\left(X^{r}\right)=\int_{0}^{\infty} x^{r}\left(b x+a x^{k}\right) e^{-\left(\frac{b}{2} x^{2}+\frac{a}{k+1} x^{k+1}\right)} d x, \quad r=1,2, \ldots
$$

Using the Taylor expansion $e^{-\frac{a}{k+1} x^{k+1}}$ and then the transformation $y=x^{2}$, we obtain $\mu_{r}=\sum_{i=0}^{\infty} \frac{(-1)^{i}}{i !}\left(\frac{a}{k+1}\right)^{i}\left[\frac{\Gamma\left(\frac{1}{2}[r+(k+1) i]+1\right)}{(b / 2)^{\frac{1}{2}[r+(k+1) i]}}+\frac{a \Gamma\left(\frac{1}{2}[r+(k+1) i+k+1]\right)}{2(b / 2)^{\frac{1}{2}[r+(k+1) i+k+1]}}\right]$

where $\Gamma($.$) is the gamma function.$

\section{Point estimation}

In reliability and survival analysis studies, the experimenter may not always obtain complete information on failure times for all experimental units. During experimentation, such situations arise because of removal or loss of items before they actually fail. However in general, such experiments are purposeful and preplanned in order to save time and cost associated with the testing. Data obtained through such experiments are called censored 
data. The two well-known censoring schemes are type-I and type-II censoring. However, these censoring schemes do not allow for removal of items other than the terminal point of the experiment (Rastogi et al. [25]). A more general censoring scheme where removal of the items is also allowed in between was introduced by Cohen [6]. This general censoring is referred to as the progressive censoring scheme and it has gained special attention in theoretical and applied statistics due to the flexibility in removing the pre-specified number of item during the test. For a comprehensive treatment of progressive censoring detailing and application of likelihood based inference under progressive censoring, see the book by Balakrishnan and Aggarwala [3].

In this study, we consider progressive type-II censoring. Suppose that $n$ units are placed on a life testing experiment. At the time of occurrence of the first failure, the $R_{1}$ out of $n-1$ surviving units are withdrawn randomly from the experiment. In a similar manner, at the time of occurrence of the second failure, the $R_{2}$ out of $n-R_{1}-2$ surviving units are withdrawn from the experiment. Finally, at the time of $m$ th $(1 \leq m \leq n)$ failure, the $R_{m}=n-m-R_{1}-\ldots-R_{m-1}$ remaining surviving units are withdrawn from the experiment. The observed sample is referred to as progressive type-II censored sample of size $m$ and the corresponding scheme is referred to as progressive type-II right censoring scheme $\left(R_{1}, \ldots, R_{m}\right)$. Note that, if $R_{1}=R_{2}=\ldots=R_{m}=0$, the progressive censoring scheme is reduced to complete sampling scheme and if $R_{1}=R_{2}=\ldots=R_{m-1}=0$ and $R_{m}=n-m$, this scheme reduces to conventional type-II censoring scheme. Statistical inference on the parameters of some distributions under progressive type-II censoring has been investigated by several authors such as Kus and Kaya [14], Jiang et al. [12], Rastogi and Tripathi [26] and Akdam et al. [1].

In this section, we obtain the MLEs and the Bayes estimates of unknown parameters $a, b$ and $k$ under SEL, LINEX and Stein loss functions based on progressively type-II censored data.

3.1. Maximum likelihood estimation. Let $\mathbf{X}=\left(X_{1: m: n}, \ldots, X_{m: m: n}\right)$ be a progressively type-II censored sample with the censoring scheme $\left(R_{1}, \ldots, R_{m}\right)$ from a continuous population with cdf $F(x)$ and pdf $f(x)$. Then, the joint pdf of this sample is given by (Balakrishnan and Aggarwala [3])

$$
f_{X_{1: m: n}, \ldots, X_{m: m: n}}\left(x_{1: m: n}, \ldots, x_{m: m: n}\right)=C \prod_{i=1}^{m} f\left(x_{i: m: n}\right)\left(1-F\left(x_{i: m: n}\right)\right)^{R_{i}}
$$

where

$$
C=n\left(n-R_{1}-1\right) \ldots\left(n-R_{1}-\ldots-R_{m-1}-m+1\right) .
$$

Substituting $F$ and $f$ from (2.1) and (2.2) in (3.1), the likelihood function is obtained as

$$
\begin{aligned}
L(b, a, k \mid \mathbf{x})= & C \prod_{i=1}^{m}\left(b x_{i: m: n}+a x_{i: m: n}^{k}\right) e^{-\frac{b}{2} \sum_{i=1}^{m} x_{i: m: n}^{2}-\frac{a}{k+1} \sum_{i=1}^{m} x_{i: m: n}^{k+1}} \\
& \times e^{-\frac{b}{2} \sum_{i=1}^{m} R_{i} x_{i: m: n}^{2}-\frac{a}{k+1} \sum_{i=1}^{m} R_{i} x_{i: m: n}^{k+1}}
\end{aligned}
$$

and hence the log-likelihood function is given by

$$
\begin{aligned}
\ell(b, a, k \mid x) \propto \sum_{i=1}^{m} \ln \left(b x_{i: m: n}+a x_{i: m: n}^{k}\right) & -\frac{b}{2} \sum_{i=1}^{m} x_{i: m: n}^{2}\left(1+R_{i}\right) \\
& -\frac{a}{k+1} \sum_{i=1}^{m} x_{i: m: n}^{k+1}\left(1+R_{i}\right) .
\end{aligned}
$$


Next, the likelihood equations of $(b, a, k)$ are obtained in the following form

$$
\begin{aligned}
\frac{\partial \ell}{\partial b} & =\sum_{i=1}^{m} \frac{x_{i: m: n}}{b x_{i: m: n}+a x_{i: m: n}^{k}}-\frac{1}{2} \sum_{i=1}^{m} x_{i: m: n}^{2}\left(1+R_{i}\right)=0, \\
\frac{\partial \ell}{\partial a}= & \sum_{i=1}^{m} \frac{x_{i: m: n}^{k}}{b x_{i: m: n}+a x_{i: m: n}^{k}}-\frac{1}{k+1} \sum_{i=1}^{m} x_{i: m: n}^{k+1}\left(1+R_{i}\right)=0, \\
\frac{\partial \ell}{\partial k} & =\sum_{i=1}^{m} \frac{a x_{i: m: n}^{k} \ln x_{i: m: n}}{b x_{i: m: n}+a x_{i: m: n}^{k}} \\
& -\frac{a}{(k+1)^{2}} \sum_{i=1}^{m} x_{i: m: n}^{k+1}\left(1+R_{i}\right)\left[(k+1) \ln x_{i: m: n}-1\right]=0 .
\end{aligned}
$$

To find the MLEs of the parameters $b, a$ and $k$, say $\hat{b}, \hat{a}$ and $\hat{k}$, we have to solve the above system of nonlinear equations (3.4)-(3.6) with respect to $b, a$ and $k$. These equations cannot be solved analytically and therefore, some numerical procedures such as Newton-Raphson (NR) method have to be used to determine the estimates. Also, to find the standard error of the MLEs of the parameters $b, a$ and $k$, we can obtain the asymptotic variance-covariance matrix of the MLEs, which need to calculate the Fisher information matrix. Unfortunately, the expected information matrix is very difficult to obtain and therefore, we compute the observed information matrix. The $3 \times 3$ observed information matrix $I$ is given by

$$
I=\left(\begin{array}{ccc}
I_{11} & I_{12} & I_{13} \\
I_{21} & I_{22} & I_{23} \\
I_{31} & I_{32} & I_{33}
\end{array}\right)
$$

where from (3.4)-(3.6) we have

$$
\begin{gathered}
I_{11}=-\frac{\partial^{2} \ell}{\partial b^{2}}=\sum_{i=1}^{m} \frac{x_{i: m: n}^{2}}{\left(b x_{i: m: n}+a x_{i: m: n}^{k}\right)^{2}}, I_{12}=-\frac{\partial^{2} \ell}{\partial b \partial a}=\sum_{i=1}^{m} \frac{x_{i: m: n}^{k+1}}{\left(b x_{i: m: n}+a x_{i: m: n}^{k}\right)^{2}}, \\
I_{13}=-\frac{\partial^{2} \ell}{\partial b \partial k}=\sum_{i=1}^{m} \frac{a x_{i: m: n}^{k+1} \ln x_{i: m: n}}{\left(b x_{i: m: n}+a x_{i: m: n}^{k}\right)^{2}}, I_{22}=-\frac{\partial^{2} \ell}{\partial a^{2}}=\sum_{i=1}^{m} \frac{x_{i: m: n}^{2 k}}{\left(b x_{i: m: n}+a x_{i: m: n}^{k}\right)^{2}}, \\
I_{23}=-\frac{\partial^{2} \ell}{\partial a \partial k}=-\sum_{i=1}^{m} \frac{b x_{i: m: n}^{k+1} \ln x_{i: m: n}}{\left(b x_{i: m: n}+a x_{i: m: n}^{k}\right)^{2}} \\
\quad+\frac{1}{(k+1)^{2}} \sum_{i=1}^{m}\left(1+R_{i}\right) x_{i: m: n}^{k+1}\left[(k+1) \ln x_{i: m: n}-1\right], \\
\quad-\frac{\partial^{2} \ell}{\partial k^{2}}=-a \sum_{i=1}^{m} \frac{b x_{i: m: n}^{k+1} \ln ^{2} x_{i: m: n}}{\left(b x_{i: m: n}+a x_{i: m: n}^{k}\right)^{2}} \\
\quad+\frac{a}{(k+1)^{3}} \sum_{i=1}^{m}\left(1+R_{i}\right) x_{i: m: n}^{k+1}\left[(k+1)^{2} \ln ^{2} x_{i: m: n}-2(k+1) \ln x_{i: m: n}+2\right] .
\end{gathered}
$$

Then, the variance-covariance matrix $\mathbf{V}$ can be approximated by the reciprocal of the observed information matrix, i.e., $\mathbf{V}=I^{-1}$. Since $\mathbf{V}$ involves the parameters $b, a$ and $k$, we replace the parameters by the corresponding MLEs in order to obtain an estimate of $\mathbf{V}$, which is denoted by

$$
\hat{V}=\left(\begin{array}{lll}
\hat{V}_{11} & \hat{V}_{12} & \hat{V}_{13} \\
\hat{V}_{21} & \hat{V}_{22} & \hat{V}_{23} \\
\hat{V}_{31} & \hat{V}_{32} & \hat{V}_{33}
\end{array}\right)=\left(\begin{array}{ccc}
\hat{I}_{11} & \hat{I}_{12} & \hat{I}_{13} \\
\hat{I}_{21} & \hat{I}_{22} & I_{23} \\
\hat{I}_{31} & \hat{I}_{32} & \hat{I}_{33}
\end{array}\right)^{-1},
$$

where $\hat{I}_{i j}$ is the $(i, j)$ th element of the observed information matrix $I$ with $b, a$ and $k$ replaced by $\hat{b}, \hat{a}$ and $\hat{k}$, respectively. 
To employ the NR algorithm, the second derivatives of the log-likelihood are required for all iterations, which may sometimes be complicated. It is also important to point out that the MLEs via the NR method are very sensitive to their initial parameter estimation value and that the NR method does not converge in all the cases. Here, we suggest to use the EM algorithm to compute the MLEs which involves solving three one-dimensional equations.

3.1.1. EM algorithm. EM algorithm, introduced by Dempster et al. [8], is a very powerful tool to handle any missing or incomplete data situation. It is an iterative method which repeatedly replaces the missing data with estimated values and updates the parameter estimates. The progressive right censoring model problem can be viewed as an incomplete data problem and then the EM algorithm may be applied to the problem of obtaining the MLEs of the parameters. First of all, we denote the observed and censored data by

$$
\mathbf{X}=\left(X_{1: m: n}, \ldots, X_{m: m: n}\right), \quad \mathbf{Z}=\left(Z_{1}, \ldots, Z_{m}\right),
$$

respectively, where $Z_{j}$ is a $1 \times R_{j}$ vector with $Z_{j}=\left(Z_{j 1}, \ldots, Z_{j R_{j}}\right)$, for $j=1, \ldots, m$. The censored data vector $\mathbf{Z}$ can be thought of as the missing data. We combine $\mathbf{X}$ and $\mathbf{Z}$ to form $\mathbf{W}$, which is the complete data set. The corresponding log-likelihood function is denoted by $\ell_{c}(\mathbf{W} ; \theta)$. For the P-LHRD, we have

$$
\begin{aligned}
\ell_{c}(\mathbf{W} ; b, a, k)= & \sum_{i=1}^{n} \ln \left(b w_{i}+a w_{i}^{k}\right)-\frac{b}{2} \sum_{i=1}^{n} w_{i}^{2}-\frac{a}{k+1} \sum_{i=1}^{n} w_{i}^{k+1} \\
= & \sum_{j=1}^{m} \ln \left(b x_{j: m: n}+a x_{j: m: n}^{k}\right)-\frac{b}{2} \sum_{j=1}^{m} x_{j: m: n}^{2}-\frac{a}{k+1} \sum_{j=1}^{m} x_{j: m: n}^{k+1} \\
& +\sum_{j=1}^{m} \sum_{\ell=1}^{R_{j}} \ln \left(b z_{j \ell}+a z_{j \ell}^{k}\right)-\frac{b}{2} \sum_{j=1}^{m} \sum_{\ell=1}^{R_{j}} z_{j \ell}^{2}-\frac{a}{k+1} \sum_{j=1}^{m} \sum_{\ell=1}^{R_{j}} z_{j \ell}^{k+1} .
\end{aligned}
$$

The MLEs of the parameters based on the complete data, using the derivatives of (3.8) with respect to $b, a$ and $k$, can be obtained by solving the equations

$$
\begin{aligned}
\frac{\partial \ell_{c}}{\partial b}= & \sum_{j=1}^{m} \frac{x_{j: m: n}}{b x_{j: m: n}+a x_{j: m: n}^{k}}-\frac{1}{2} \sum_{j=1}^{m} x_{j: m: n}^{2} \\
& +\sum_{j=1}^{m} \sum_{\ell=1}^{R_{j}} \frac{z_{j \ell}}{b z_{j \ell}+a z_{j \ell}^{k}}-\frac{1}{2} \sum_{j=1}^{m} \sum_{\ell=1}^{R_{j}} z_{j \ell}^{2}=0,
\end{aligned}
$$

$$
\begin{aligned}
\frac{\partial \ell_{c}}{\partial a}= & \sum_{j=1}^{m} \frac{x_{j: m: n}^{k}}{b x_{j: m: n}+a x_{j: m: n}^{k}}-\frac{1}{k+1} \sum_{j=1}^{m} x_{j: m: n}^{k+1} \\
& +\sum_{j=1}^{m} \sum_{\ell=1}^{R_{j}} \frac{z_{j \ell}^{k}}{b z_{j \ell}+a z_{j \ell}^{k}}-\frac{1}{k+1} \sum_{j=1}^{m} \sum_{\ell=1}^{R_{j}} z_{j \ell}^{k+1}=0,
\end{aligned}
$$

$$
\frac{\partial \ell_{c}}{\partial k}=\sum_{j=1}^{m} \frac{x_{j: m: n}^{k} \ln x_{j: m: n}}{b x_{j: m: n}+a x_{j: m: n}^{k}}-\frac{1}{(k+1)^{2}} \sum_{j=1}^{m} x_{j: m: n}^{k+1}\left[(k+1) \ln x_{j: m: n}-1\right]
$$

$$
+\sum_{j=1}^{m} \sum_{\ell=1}^{R_{j}} \frac{z_{j \ell}^{k} \ln z_{j \ell}}{b z_{j \ell}+a z_{j \ell}^{k}}-\frac{1}{(k+1)^{2}} \sum_{j=1}^{m} \sum_{\ell=1}^{R_{j}} z_{j \ell}^{k+1}\left[(k+1) \ln z_{j \ell}-1\right]=0,
$$

simultaneously. 
The EM algorithm consists of two steps: The E-step and the M-step. In the expectation, or E-step, the missing data are estimated given the observed data and current estimate of the model parameters, say $\theta_{(h)}$. Then, the E-step of the algorithm requires the computation of

$$
Q\left(\theta \mid \theta_{(h)}\right)=E\left(\ell_{c}(\mathbf{W} ; \theta) \mid \mathbf{X}=\mathbf{x}, \theta_{(h)}\right),
$$

which mainly involves the computation of the conditional expectation of functions of $\mathbf{Z}$ conditional on the observed values $\mathbf{X}$ and the current value of the parameters. Therefore, in order to facilitate the EM algorithm, the conditional distribution of $\mathbf{Z}$, conditional on $\mathbf{X}$ and the current value of the parameters, needs to be determined. Given $X_{1: m: n}=x_{1: m: n}, X_{2: m: n}=x_{2: m: n} \ldots, X_{j: m: n}=x_{j: m: n}$, the conditional distribution of $Z_{j l}$, $l=1, \ldots, R_{j}$ is given by

$$
\begin{aligned}
& f_{Z \mid X}\left(z_{j l} \mid X_{1: m: n}=x_{1: m: n}, X_{2: m: n}=x_{2: m: n}, \ldots, X_{j: m: n}=x_{j: m: n}\right) \\
& \quad=f_{Z \mid X}\left(z_{j l} \mid X_{j: m: n}=x_{j: m: n}\right)=\frac{f_{W}\left(z_{j l}\right)}{1-F_{W}\left(x_{j: m: n}\right)}, \quad z_{j l}>x_{j: m: n},
\end{aligned}
$$

and $Z_{j l}$ and $Z_{j p}, l \neq p$, are conditionally independent given $X_{j: m: n}=x_{j: m: n}$ (see $\mathrm{Ng}$ et al. [24]). For the P-LHRD, using (3.12), we have

$$
\begin{aligned}
f_{Z \mid X}\left(z_{j l} \mid x_{j: m: n}, b, a, k\right) & =\left(b z_{j l}+a z_{j l}^{k}\right) \\
& \times e^{-\frac{b}{2} z_{j l}^{2}-\frac{a}{k+1} z_{j l}^{k+1}+\frac{b}{2} x_{j: m: n}^{2}+\frac{a}{k+1} x_{j: m: n}^{k+1}, z_{j l}>x_{j: m: n} .}
\end{aligned}
$$

Using (3.13), the required conditional expectations in the EM algorithm are derived in the Appendix.

In the maximization or M-step of the EM algorithm, the value of $\theta$ which maximizes $Q\left(\theta \mid \theta_{(h)}\right)$ will be used as the next estimate of $\theta_{(h+1)}$. In the M-step of the $(h+1)$ th iteration of the EM algorithm, using (3.9)-(3.11), the value of $b_{(h+1)}$ is first obtained by solving the equation

$$
\begin{gathered}
\sum_{j=1}^{m} \frac{x_{j: m: n}}{b_{(h+1)} x_{j: m: n}+a_{(h)} x_{j: m: n}^{k_{(h)}}}+\sum_{j=1}^{m} R_{j} E\left(\frac{Z_{j}}{b Z_{j}+a Z_{j}^{k}} \mid x_{j: m: n}, b_{(h)}, a_{(h)}, k_{(h)}\right) \\
-\frac{1}{2} \sum_{j=1}^{m} x_{j: m: n}^{2}-\frac{1}{2} \sum_{j=1}^{m} R_{j} E\left(Z_{j}^{2} \mid x_{j: m: n}, b_{(h)}, a_{(h)}, k_{(h)}\right)=0,
\end{gathered}
$$

then $a_{(h+1)}$ is obtained by solving the equation

$$
\begin{array}{cc}
\sum_{j=1}^{m} & \frac{x_{j: m: n}^{k_{(h)}}}{b_{(h+1)} x_{j: m: n}+a_{(h+1)} x_{j: m: n}^{k_{(h)}}}+\sum_{j=1}^{m} R_{j} E\left(\frac{Z_{j}^{k}}{b Z_{j}+a Z_{j}^{k}} \mid x_{j: m: n}, b_{(h+1)}, a_{(h)}, k_{(h)}\right) \\
& -\frac{1}{k_{(h)}+1} \sum_{j=1}^{m} x_{j: m: n}^{k_{(h)}+1}-\frac{1}{k_{(h)}+1} \sum_{j=1}^{m} R_{j} E\left(Z_{j}^{k+1} \mid x_{j: m: n}, b_{(h+1)}, a_{(h)}, k_{(h)}\right)=0,
\end{array}
$$

and finally, $k_{(h+1)}$ is obtained by solving the equation

$$
\begin{aligned}
& \sum_{j=1}^{m} \frac{x_{j: m: n}^{k_{(h+1)}} \ln x_{j: m: n}}{b_{(h+1)} x_{j: m: n}+a_{(h+1)} x_{j: m: n}^{k_{(h+1)}}}+\sum_{j=1}^{m} R_{j} E\left(\frac{Z_{j}^{k} \ln Z_{j}}{b Z_{j}+a Z_{j}^{k}} \mid x_{j: m: n}, b_{(h+1)}, a_{(h+1)}, k_{(h)}\right) \\
& -\frac{1}{\left(k_{(h+1)}+1\right)^{2}} \sum_{j=1}^{m} x_{j: m: n}^{k_{(h+1)}+1}\left[\left(k_{(h+1)}+1\right) \ln x_{j: m: n}-1\right] \\
& \quad-\frac{1}{\left(k_{(h+1)}+1\right)^{2}} \sum_{j=1}^{m} R_{j} E\left(Z_{j}^{k+1}\left[(k+1) \ln Z_{j}-1\right] \mid x_{j: m: n}, b_{(h+1)}, a_{(h+1)}, k_{(h)}\right)=0,
\end{aligned}
$$


where the desired expectations are computed from (7.2)-(7.7). The MLEs of $(b, a, k)$ can be obtained by repeating the E-step and M-step until convergence occurs. Although the $\mathrm{EM}$ algorithm is relatively robust against the initial values, it may not converge when initial values are far from good ones. A reasonable starting value is the estimates of the parameters based on the pseudo-complete sample by replacing the censored observations $Z_{j}$ by $X_{j: m: n}, j=1,2, \ldots, m$ (Ng et al., [22]).

3.2. Bayesian estimation. In this subsection, we discuss Bayesian estimation of the parameters of the P-LHRD based on progressively type-II censored sample. An important element of Bayesian analysis is the selection of a loss function $L(\theta, \delta)$, where $\delta$ is a decision rule based on the data. One of the most commonly used loss function is squared error loss (SEL) function, $L(\theta, \delta(X))=(\delta(X)-\theta)^{2}$. The SEL function is a symmetric loss function that assigns equal losses to overestimation and underestimation. However, such a restriction may not be practical because in some cases overestimation may by more serious than underestimation or vice versa. For example, overestimation is usually more serious than underestimation in the estimation of reliability and failure rate functions. In these cases, the use of symmetric loss functions may be inappropriate. On the other hand, the use of an asymmetric loss function which assigns greater importance to overestimation or underestimation may be more appropriate.

A useful alternative to the SEL function is convex but asymmetric loss function proposed by Varian [34], which is known as LINEX loss function and is defined as

$$
L(\theta, \delta)=e^{c(\delta-\theta)}-c(\delta-\theta)-1, \quad c \neq 0
$$

where $\theta$ is the unknown parameter. The shape parameter $c$ is known and gives the degree of asymmetry. If $c>0$, the overestimation is more serious than underestimation and if $c<0$, underestimation is more serious than overestimation. If $c$ close to zero, the LINEX loss is approximately SEL and therefore almost symmetric. Under the LINEX loss function (3.17), the Bayes estimator of $\theta$ that minimizes the posterior risk $E[L(\theta, \delta(X)) \mid X]$ is given by

$$
\delta_{B L}(X)=-\frac{1}{c} \ln E\left(e^{-c \theta} \mid X\right)
$$

provided that the expectation exists and is finite.

Another useful asymmetric loss function is the Stein loss function which is also known as entropy loss function. This loss function has the form

$$
L(\theta, \delta)=\frac{\delta}{\theta}-\ln \frac{\delta}{\theta}-1
$$

where $\theta$ is the unknown parameter. This loss is a convex function of $\delta$ and more penalized underestimation than overestimation. Under this loss function the Bayes estimator $\delta_{B S T}(X)$ that minimizes the posterior risk $E[L(\theta, \delta(X)) \mid X]$ is given by

$$
\delta_{B S T}(X)=\left\{E\left(\frac{1}{\theta} \mid X\right)\right\}^{-1}
$$

provided that the expectation exists. For more details and estimation under entropy loss function, see Parsian and Nematollahi [24].

To obtain Bayes estimates, we assume that $b, a$ and $(k+1)$ have independent gamma priors as $\operatorname{Gamma}\left(\alpha_{1}, \beta_{1}\right)$, Gamma $\left(\alpha_{2}, \beta_{2}\right)$ and $\operatorname{Gamma}\left(\alpha_{3}, \beta_{3}\right)$, respectively, with pdfs given by

$$
\begin{aligned}
& \pi\left(b \mid \alpha_{1}, \beta_{1}\right)=\frac{\beta_{1}^{\alpha_{1}} b^{\alpha_{1}-1} e^{-\beta_{1} b}}{\Gamma\left(\alpha_{1}\right)}, \quad b>0, \quad \alpha_{1}, \beta_{1}>0, \\
& \pi\left(a \mid \alpha_{2}, \beta_{2}\right)=\frac{\beta_{2}^{\alpha_{2}} a^{\alpha_{2}-1} e^{-\beta_{2} a}}{\Gamma\left(\alpha_{2}\right)}, \quad a>0, \quad \alpha_{2}, \beta_{2}>0,
\end{aligned}
$$




$$
\pi\left(k \mid \alpha_{3}, \beta_{3}\right)=\frac{\beta_{3}^{\alpha_{3}}(k+1)^{\alpha_{3}-1} e^{-\beta_{3}(k+1)}}{\Gamma\left(\alpha_{3}\right)}, \quad k>-1, \quad \alpha_{3}, \beta_{3}>0,
$$

where the hyperparameters $\alpha_{1}, \alpha_{2}$ and $\alpha_{3}$ are known but $\beta_{1}, \beta_{2}$ and $\beta_{3}$ are unknown. Here, we assign conjugate prior distributions to the hyperparameters $\beta_{1}, \beta_{2}$ and $\beta_{3}$ as $\operatorname{Gamma}\left(\eta_{1}, \gamma_{1}\right), \operatorname{Gamma}\left(\eta_{2}, \gamma_{2}\right)$ and $\operatorname{Gamma}\left(\eta_{3}, \gamma_{3}\right)$, respectively. Then the hierarchical prior density function of $b$ is given by

$$
\begin{aligned}
\pi(b)=\int_{0}^{\infty} \pi\left(b \mid \alpha_{1}, \beta_{1}\right) \pi\left(\beta_{1}\right) d \beta_{1} & =\frac{\gamma_{1}^{\eta_{1}} b^{\alpha_{1}-1}}{\Gamma\left(\alpha_{1}\right) \Gamma\left(\eta_{1}\right)} \int_{0}^{\infty} \beta_{1}^{\alpha_{1}+\eta_{1}-1} e^{-\left(b+\gamma_{1}\right) \beta_{1}} d \beta_{1} \\
& =\frac{\Gamma\left(\alpha_{1}+\eta_{1}\right)}{\Gamma\left(\alpha_{1}\right) \Gamma\left(\eta_{1}\right)} \frac{\gamma_{1}^{\eta_{1}} b^{\alpha_{1}-1}}{\left(b+\gamma_{1}\right)^{\alpha_{1}+\eta_{1}}}, \quad b>0 .
\end{aligned}
$$

Similarly, the hierarchical prior density functions of $a$ and $k$ are given by

$$
\begin{gathered}
\pi(a)=\frac{\Gamma\left(\alpha_{2}+\eta_{2}\right)}{\Gamma\left(\alpha_{2}\right) \Gamma\left(\eta_{2}\right)} \frac{\gamma_{2}^{\eta_{2}} a^{\alpha_{2}-1}}{\left(a+\gamma_{2}\right)^{\alpha_{2}+\eta_{2}}}, \quad a>0, \\
\pi(k)=\frac{\Gamma\left(\alpha_{3}+\eta_{3}\right)}{\Gamma\left(\alpha_{3}\right) \Gamma\left(\eta_{3}\right)} \frac{\gamma_{3}^{\eta_{3}}(k+1)^{\alpha_{3}-1}}{\left(k+\gamma_{3}+1\right)^{\alpha_{3}+\eta_{3}}}, \quad k>-1 .
\end{gathered}
$$

Now, using (3.2) and (3.21)-(3.23), the joint posterior density function of $b, a$ and $k$ can be written as

$$
\begin{aligned}
& \pi(b, a, k \mid \mathbf{x}) \propto \pi(b) \pi(a) \pi(k) L(b, a, k \mid \mathbf{x}) \\
& \propto \frac{b^{\alpha_{1}-1}}{\left(b+\gamma_{1}\right)^{\alpha_{1}+\eta_{1}}} \frac{a^{\alpha_{2}-1}}{\left(a+\gamma_{2}\right)^{\alpha_{2}+\eta_{2}}} \frac{(k+1)^{\alpha_{3}-1}}{\left(k+\gamma_{3}+1\right)^{\alpha_{3}+\eta_{3}}} \prod_{i=1}^{m}\left(b+a x_{i: m: n}^{k-1}\right) \\
& \times e^{-\frac{b}{2} \sum_{i=1}^{m}\left(1+R_{i}\right) x_{i: m: n}^{2}-\frac{a}{k+1} \sum_{i=1}^{m}\left(1+R_{i}\right) x_{i: m: n}^{k+1} .}
\end{aligned}
$$

The expression for $\pi(b, a, k \mid \mathbf{x})$ in $(3.24)$ can not be written in a closed form because the integration of the joint posterior density is not easy to perform. Therefore, we need a simulation technique to compute the Bayes estimate of the parameters and their corresponding credible interval. We adopt the Gibbs sampling technique which use the posterior distributions of each parameter conditional on all others (see Gelfand and Smith [10]). The full conditional distributions of $b, a$ and $k$ can be obtained as follows

$$
\begin{aligned}
\pi(b \mid a, k, \mathbf{x}) & \propto \frac{b^{\alpha_{1}-1}}{\left(b+\gamma_{1}\right)^{\alpha_{1}+\eta_{1}}} \prod_{i=1}^{m}\left(b+a x_{i: m: n}^{k-1}\right) e^{-\frac{b}{2} \sum_{i=1}^{m}\left(1+R_{i}\right) x_{i: m: n}^{2}}, \\
\pi(a \mid b, k, \mathbf{x}) & \propto \frac{a^{\alpha_{2}-1}}{\left(a+\gamma_{2}\right)^{\alpha_{2}+\eta_{2}}} \prod_{i=1}^{m}\left(b+a x_{i: m: n}^{k-1}\right) e^{-\frac{a}{k+1} \sum_{i=1}^{m}\left(1+R_{i}\right) x_{i: m: n}^{k+1}}, \\
\pi(k \mid b, a, \mathbf{x}) & \propto \frac{(k+1)^{\alpha_{3}-1}}{\left(k+\gamma_{3}+1\right)^{\alpha_{3}+\eta_{3}}} \prod_{i=1}^{m}\left(b+a x_{i: m: n}^{k-1}\right) e^{-\frac{a}{k+1} \sum_{i=1}^{m}\left(1+R_{i}\right) x_{i: m: n}^{k+1}} .
\end{aligned}
$$

Since the full conditional distributions for $b, a$ and $k$ do not have explicit expressions, it is not possible to sample directly by standard methods. To do this, we use the MetropolisHastings algorithm into the Gibbs sampling algorithm as explained by Tierney [33]. Therefore, the algorithm of Gibbs sampling is described as follows

Step 1. Start with an initial guess $\left(b^{(0)}, a^{(0)}, k^{(0)}\right)$ and set $\mathrm{t}=1$.

Step 2. Using Metropolis-Hastings method, generate $b^{(t)}$ from $\pi\left(b \mid a^{(t-1)}, k^{(t-1)}, \mathbf{x}\right)$ with the proposal distribution as

$$
q(b) \propto N\left(b^{(t-1)}, C_{b} \hat{V}_{11}\right) I(b>0),
$$

where $C_{b}$ is a scaling factor, $\hat{V}_{11}$ is given in (3.7) and $N\left(\mu, \sigma^{2}\right)$ is the normal distribution with the parameters $\mu$ and $\sigma^{2}$. 
Step 3. Using Metropolis-Hastings method, generate $a^{(t)}$ from $\pi\left(a \mid b^{(t)}, k^{(t-1)}, \mathbf{x}\right)$ with the proposal distribution as

$$
q(a) \propto N\left(a^{(t-1)}, C_{a} \hat{V}_{22}\right) I(a>0),
$$

where $C_{a}$ is a scaling factor and $\hat{V}_{22}$ is given in (3.7).

Step 4. Using Metropolis-Hastings method, generate $k^{(t)}$ from $\pi\left(k \mid b^{(t)}, a^{(t)}, \mathbf{x}\right)$ with the proposal distribution as

$$
q(k) \propto N\left(k^{(t-1)}, C_{k} \hat{V}_{33}\right) I(k>-1),
$$

where $C_{k}$ is a scaling factor and $\hat{V}_{33}$ is given in (3.7).

Step 5. Set $\mathrm{t}=\mathrm{t}+1$.

Step 6. Repeat Steps $2-5, \mathrm{~N}$ times, and obtain the posterior sample $\left(b^{(t)}, a^{(t)}, k^{(t)}\right)$, $t=1, \ldots, N$.

Now, we can use this sample to compute the Bayes estimate for the parameters $b, a$ and $k$. The approximate Bayes estimate of the parameters under the SE, the LINEX and the Stein loss functions are given as

$$
\begin{gathered}
\tilde{\theta}_{B S}=\hat{E}(\theta \mid X)=\frac{1}{N-M} \sum_{i=M+1}^{N} \theta^{(i)}, \\
\tilde{\theta}_{B L}=-\frac{1}{c} \ln \hat{E}\left(e^{-c \theta} \mid X\right)=-\frac{1}{c} \ln \left(\frac{1}{N-M} \sum_{i=M+1}^{N} e^{-c \theta^{(i)}}\right), \\
\tilde{\theta}_{B S T}=\left\{\hat{E}\left(\frac{1}{\theta} \mid X\right)\right\}^{-1}=\left\{\frac{1}{N-M} \sum_{i=M+1}^{N} \frac{1}{\theta^{(i)}}\right\}^{-1},
\end{gathered}
$$

respectively, where $M$ is the burn-in period and $\theta$ can be each of the parameters $b, a$ and $k$. Note that, the Stein loss function is suitable for estimation of the parameters with positive values. So, in what follows, we will not use it for estimation of the parameter $k$.

\section{Interval estimation}

In this section, we discuss interval estimation of the parameters of the P-LHRD based on progressively type-II censored sample.

4.1. Asymptotic confidence interval. As discussed earlier, the MLEs of the unknown parameters $b, a$ and $k$ can not be obtained in closed forms. Therefore, it is not easy to derive the exact distributions of the MLEs. In this subsection, we derive the approximate confidence intervals of these parameters based on the asymptotic distributions of their MLEs. The asymptotic distribution of the MLE of the parameters $b, a$ and $k$ is given by (see Miller [19])

$$
\left(\begin{array}{c}
\hat{b} \\
\hat{a} \\
\hat{k}
\end{array}\right) \sim N\left(\left(\begin{array}{c}
b \\
a \\
k
\end{array}\right), \hat{V}\right),
$$

where $\hat{V}$ is given in (3.7). Now, using (4.1), the $100(1-\alpha) \%$ approximate confidence intervals for the parameters $b, a$ and $k$ are given by

$$
\hat{b} \pm z_{\alpha / 2} \sqrt{\hat{V}_{11}}, \quad \hat{a} \pm z_{\alpha / 2} \sqrt{\hat{V}_{22}}, \quad \hat{k} \pm z_{\alpha / 2} \sqrt{\hat{V}_{33}}
$$

respectively, where $z_{\alpha / 2}$ is the $(1-\alpha / 2)$ quantile of the standard normal distribution. 
4.2. Bootstrap confidence intervals. When the effective sample size $m$ is small, the normal approximations may not work well. In such situations, resampling techniques such as the bootstrap may provide more accurate approximate confidence intervals. In this subsection, we propose two confidence intervals for the parameters $b, a$ and $k$ based on the parametric bootstrap methods as suggested by Efron and Tibshirani [9]: the percentile bootstrap (Boot-p) confidence interval and the student's $t$ bootstrap (Boott) confidence interval. Firstly, by using the algorithm suggested in Balakrishnan and Sandhu [4], we perform the following steps to generate progressively type-II censored samples from P-LHRD:

Step 1. Generate $m$ independent samples $U_{1}, U_{2}, \ldots, U_{m}$ from the standard uniform distribution $U(0,1)$.

Step 2. For given values of the progressive censoring scheme $\left(R_{1}, R_{2}, \ldots, R_{m}\right)$, set

$$
V_{i}=U_{i}^{1 /\left(i+\sum_{j=m-i+1}^{m} R_{j}\right)}, \quad i=1,2, \ldots, m .
$$

Step 3. Set $U_{i: m: n}=1-V_{m} V_{m-1} \ldots V_{m-i+1}$ for $i=1,2, \ldots, m$. Then $U_{1: m: n}, U_{2: m: n}$, $\ldots, U_{m: m: n}$ is a progressively type-II censored sample of size $m$ from $U(0,1)$ distribution.

Step 4. Finally, for given values of the parameters $b, a$ and $k$, set

$$
\frac{b}{2} X_{i: m: n}^{2}+\frac{a}{k+1} X_{i: m: n}^{k+1}+\ln \left(1-U_{i: m: n}\right)=0, \quad i=1,2, \ldots, m .
$$

Then from (2.1) $X_{1: m: n}, X_{2: m: n}, \ldots, X_{m: m: n}$ is the required progressively type-II censored sample from the P-LHRD.

Now, we use the following steps to generate parametric bootstrap samples.

Step 1. Compute $\hat{b}, \hat{a}$ and $\hat{k}$, the MLEs of the parameters $b, a$ and $k$ based on the progressive type-II censored samples $\mathbf{x}=\left(x_{1: m: n}, \ldots, x_{m: m: n}\right)$.

Step 2. Generate the bootstrap progressive type-II censored samples $\mathbf{x}^{*}=\left(x_{1: m: n}^{*}, \ldots, x_{m: m: n}^{*}\right)$ from the P-LHRD with the parameters $\hat{b}, \hat{a}$ and $\hat{k}$ by using the above proposed algorithm. By using these data, we compute the bootstrap estimates say, $\hat{b}^{*}, \hat{a}^{*}$ and $\hat{k}^{*}$.

Step 3. Repeat step 2, $B$ times to obtain a set of bootstrap progressive type-II censored samples of $b, a$ and $k$, say $\left(\hat{b}_{1}^{*}, \ldots, \hat{b}_{B}^{*}\right),\left(\hat{a}_{1}^{*}, \ldots, \hat{a}_{B}^{*}\right)$ and $\left(\hat{k}_{1}^{*}, \ldots, \hat{k}_{B}^{*}\right)$.

Using the above bootstrap samples, we obtain two different bootstrap confidence intervals for the parameter $\theta$ (in our case, $\theta$ could be $b, a$ or $k$ ) based on the following methods:

(I) Boot-p method

Let $G(x)=\operatorname{Pr}\left(\hat{\theta}^{*} \leq x\right)$ be the cdf of $\hat{\theta}^{*}$. Define $\hat{\theta}_{B o o t}(x)=G^{-1}(x)$ for a given $x$. Then the $100(1-\alpha) \%$ bootstrap percentile interval for $\theta$ is defined by

$$
\left(\hat{\theta}_{\text {Boot }}\left(\frac{\alpha}{2}\right), \hat{\theta}_{\text {Boot }}\left(1-\frac{\alpha}{2}\right)\right)
$$

that is, just use the $\alpha / 2$ and $(1-\alpha / 2)$ quantiles of the bootstrap sample $\hat{\theta_{1}^{*}}, \ldots, \hat{\theta_{B}^{*}}$.

(II) Boot-t method

Let

$$
T_{b}^{*}=\frac{\left(\hat{\theta}_{i}^{*}-\hat{\theta}\right)}{\hat{s} e_{i}^{*}}, \quad i=1,2, \ldots, B
$$


where $\hat{s} e_{i}^{*}$ is an estimate of the standard error of $\hat{\theta}_{i}^{*}$ and can be replaced by its asymptotic standard error. Then the $100(1-\alpha) \%$ bootstrap student's $t$ interval is given by

$$
\left(\hat{\theta}-t_{1-\alpha / 2}^{*} \hat{s} e_{b o o t}, \hat{\theta}-t_{\alpha / 2}^{*} \hat{s} e_{b o o t}\right)
$$

where $t_{\alpha}^{*}$ is the $\alpha$ quantile of $T_{1}^{*}, \ldots, T_{B}^{*}$ and $\hat{s} e_{b o o t}$ is the bootstrap estimate of the standard error based on $\hat{\theta_{1}^{*}}, \ldots, \hat{\theta_{B}^{*}}$.

4.3. HPD credible interval. We apply the posterior sample $\left(b^{(t)}, a^{(t)}, k^{(t)}\right), t=1, \ldots, N$, generated by the algorithm described in Subsection 3.2, to construct the $100(1-\gamma) \%$ HPD credible intervals for the parameters of the P-LHRD. We use the method of Chen and Shao [5] and construct the HPD interval as follow

$$
\left(\theta_{\left[\frac{\gamma}{2} N\right]}, \theta_{\left[\left(1-\frac{\gamma}{2}\right) N\right]}\right)
$$

where $\theta_{\left[\frac{\gamma}{2} N\right]}$ and $\theta_{\left[\left(1-\frac{\gamma}{2}\right) N\right]}$ are the $\left[\frac{\gamma}{2} N\right]$-th smallest integer and the $\left[\left(1-\frac{\gamma}{2}\right) N\right]$-th smallest integer of $\left\{\theta^{(t)}, t=M+1, M+2, \ldots, N\right\}$, respectively.

\section{Application}

In this section, we analyze a real data set to demonstrate the performance of the PLHRD in practice and compare its goodness-of-fit with other distributions using KolmogorovSmirnov (K-S) statistic. We consider the real data consist of failure times of the air conditioning system of an airplane (in hours), which are obtained from Linhart and Zucchini [17] and are presented in Table 1. Tian et al. [32] have fitted a new four-parameter generalized linear exponential distribution (NGLED) to this data and have compared the results of fitting the NGLED with some of the three-parameter distributions such as the exponentiated Weibull distribution (EWD), the generalized linear failure rate distribution (GLFRD) and the modified Weibull distribution (MWD).

Table 1. Data set (failure times of the air conditioning system)

\begin{tabular}{ccccccccccccccc}
\hline 1 & 3 & 5 & 7 & 11 & 11 & 11 & 12 & 14 & 14 & 14 & 16 & 16 & 20 & 21 \\
23 & 42 & 47 & 52 & 62 & 71 & 71 & 87 & 90 & 95 & 120 & 120 & 225 & 246 & 261 \\
\hline
\end{tabular}

First, we fit the P-LHRD to this data set and compare it with its sub-models and the distributions reported in Tian et al. [32]. The MLEs of the unknown parameters are obtained and then the values of Kolmogorov-Smirnov (K-S) statistics and their corresponding $p$-values are calculated. The results are reported in Table 2 . It is clear from Table 2 that P-LHRD has the highest $p$-value of K-S test statistic. Thus, we observe that P-LHRD yields a better fit than LHRD, PHRD, EWD, GLFRD, MWD and NGLED. Moreover, we plot the empirical cdf versus fitted cdfs and the histogram of the data versus fitted pdfs in Figure 2. These figures support our conclusion.

It is important here to note that EWD, GLFRD and MWD are three-parameter distributions with the complicated hazard rate functions. Clearly, it is merit of the PLHRD that provide a better fit than other three-parameter distributions while its hazard rate function is very simple and flexible. 
Table 2. MLEs of parameters (standard errors in parentheses), K-S statistic and corresponding $p$-value

\begin{tabular}{|c|c|c|c|c|c|c|}
\hline Distribution & & Estimates & & & K-S statistic & $p$-value \\
\hline $\operatorname{LHRD}(b, a)$ & $\begin{array}{l}6 \times 10^{-6} \\
(0.0030)\end{array}$ & $\begin{array}{c}0.0164 \\
(0.0004)\end{array}$ & - & - & 0.2179 & 0.1159 \\
\hline $\operatorname{PHRD}(a, k)$ & $\begin{array}{c}0.0215 \\
(0.0149)\end{array}$ & $\begin{array}{c}-0.0532 \\
(0.2083)\end{array}$ & - & - & 0.1761 & 0.3103 \\
\hline $\operatorname{P}-\operatorname{LHRD}(b, a, k)$ & $\begin{array}{l}2 \times 10^{-5} \\
(0.0001)\end{array}$ & $\begin{array}{c}0.0377 \\
(0.0154)\end{array}$ & $\begin{array}{c}-0.2671 \\
(0.1484)\end{array}$ & - & 0.1290 & 0.7005 \\
\hline $\operatorname{EWD}(\alpha, \beta, \gamma)$ & $\begin{array}{c}0.8384 \\
(0.2617)\end{array}$ & $\begin{array}{c}0.0161 \\
(0.0283)\end{array}$ & $\begin{array}{c}0.9832 \\
(0.3034)\end{array}$ & - & 0.1728 & 0.3322 \\
\hline $\operatorname{GLFRD}(\alpha, \lambda, \beta)$ & $\begin{array}{c}0.8064 \\
(0.1858)\end{array}$ & $\begin{array}{c}0.0144 \\
(0.0131)\end{array}$ & $\begin{array}{l}4 \times 10^{-7} \\
(0.0006)\end{array}$ & - & 0.1729 & 0.3312 \\
\hline $\operatorname{MWD}(\lambda, \beta, \gamma)$ & $\begin{array}{l}6 \times 10^{-5} \\
(0.0016)\end{array}$ & $\begin{array}{c}0.0214 \\
(0.0384)\end{array}$ & $\begin{array}{c}0.9466 \\
(0.2784)\end{array}$ & - & 0.1918 & 0.2198 \\
\hline $\operatorname{NGLED}(\alpha, \lambda, \beta, \gamma)$ & $\begin{array}{c}0.8348 \\
(0.1986)\end{array}$ & $\begin{array}{c}0.0055 \\
(0.0084)\end{array}$ & $\begin{array}{c}0.0107 \\
(0.0647)\end{array}$ & $\begin{array}{c}0.9718 \\
(0.3032)\end{array}$ & 0.1708 & 0.3455 \\
\hline
\end{tabular}
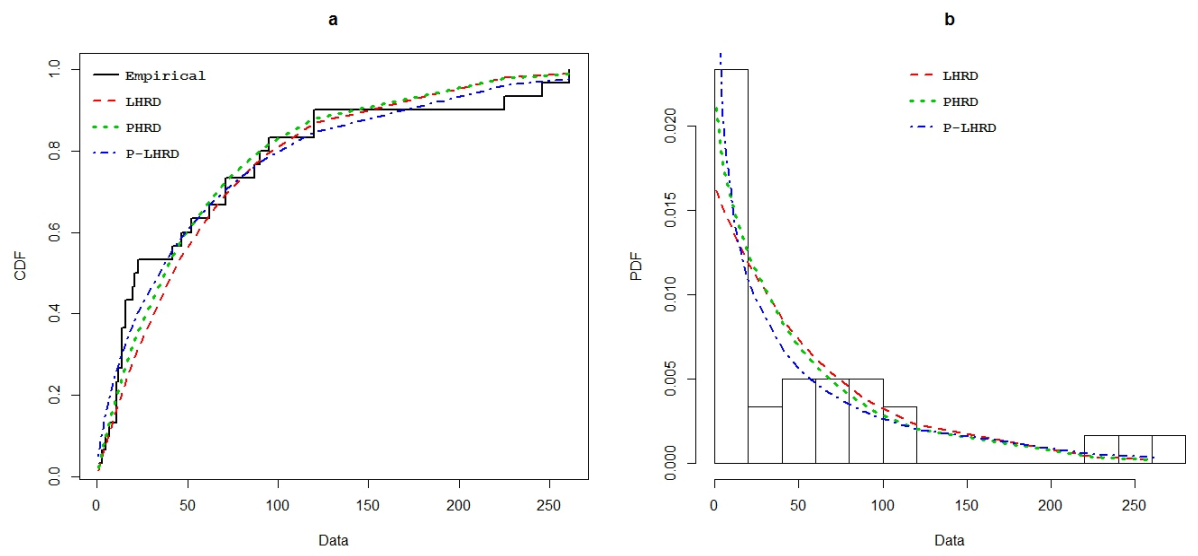

Figure 2. (a) The empirical cdf versus fitted cdfs. (b) Histogram of the data versus fitted pdfs.

For illustrative purposes, we have generated a progressively type-II censored sample of size $m=20$ from above data set with respect to the censoring scheme $\left(R_{1}=\ldots=\right.$ $\left.R_{8}=0, R_{9}=\ldots=R_{13}=1, R_{14}=0, R_{15}=3, R_{16}=\ldots=R_{19}=0, R_{20}=2\right)$. The generated data are as follows:

$1,3,5,7,11,11,12,14,16,20,21,47,52,62,71,87,120,120,225,246$.

For these progressively censored data, we obtain the MLEs of the parameters with initial values $b_{(0)}=0.00002, a_{(0)}=0.0377$ and $k_{(0)}=-0.2671$, which are the estimates of the parameters based on the complete data. The MLEs of the parameters $b, a$ and $k$ using the EM algorithm from (3.14)-(3.16) are given as

$$
\hat{b}=5 \times 10^{-7}, \quad \hat{a}=0.02242, \quad \hat{k}=-0.17253
$$


with the corresponding standard errors (Se) as

$$
S e(\hat{b})=0.0001, \quad S e(\hat{a})=0.0062, \quad S e(\hat{k})=0.0003
$$

Also, the MLEs via the NR algorithm from (3.4)-(3.6) yield the same results with the EM algorithm.

Substituting the MLE of the unknown parameters in (3.7), we obtain the estimate of the variance-covariance matrix $\hat{V}$ as

$$
\hat{V}=\left(\begin{array}{ccc}
8.04 \times 10^{-10} & -1.03 \times 10^{-7} & -5.54 \times 10^{-9} \\
-1.03 \times 10^{-7} & 3.83 \times 10^{-5} & 2.05 \times 10^{-6} \\
-5.54 \times 10^{-9} & 2.05 \times 10^{-6} & 1.11 \times 10^{-7}
\end{array}\right)
$$

Therefore, the approximate $95 \%$ confidence intervals of the parameters $b, a$ and $k$ using $(4.2)$ are given as $(-0.00005,0.00006),(0.01028,0.03455)$ and $(-0.17318,-0.17187)$, respectively. Based on 1000 bootstrap samples, the $95 \%$ Boot-p confidence intervals of the parameters $b, a$ and $k$ from (4.3) are obtained as $\left(3.07 \times 10^{-7}, 3.07 \times 10^{-5}\right),(0.0126$, $0.0695)$ and $(-0.5083,0.0344)$, respectively. Also, the $95 \%$ Boot-t confidence intervals of the parameters $b, a$ and $k$ from $(4.4)$ are obtained as $(-0.0050,0.0002),(-0.0069,0.0731)$ and $(-13.38,20.89)$, respectively.

To obtain Bayes estimates of the parameters $b, a$ and $k$, we used small values of the hyperparameters as $\left(\alpha_{1}, \eta_{1}, \gamma_{1}\right)=(0.5,0.25,0.75),\left(\alpha_{2}, \eta_{2}, \gamma_{2}\right)=(0.75,0.5,1)$ and $\left(\alpha_{3}, \eta_{3}, \gamma_{3}\right)=(1,0.25,0.75)$ to reflect little prior information. We computed the Bayes estimates and 95\% HPD credible intervals of the parameters based on $N=10000 \mathrm{MCMC}$ samples and discard the first $M=2000$ values as burn-in period. The simulated values and Histogram of the parameters $b, a$ and $k$ generated by the algorithm of Gibbs sampling are plotted in Figure 3. Based on these simulated values, the Bayes estimate of the parameters $b, a$ and $k$ under the SEL function using (3.28) are computed as

$$
\tilde{b}_{B S}=0.00005, \quad \tilde{a}_{B S}=0.03269, \quad \tilde{k}_{B S}=-0.41309 .
$$

From (3.29), the Bayes estimate of the parameters $b, a$ and $k$ under the LINEX loss function for $c=2$ are given as

$$
\tilde{b}_{B L}=0.00005, \quad \tilde{a}_{B L}=0.03251, \quad \tilde{k}_{B L}=-0.43293
$$

and for $c=-1$ are given as

$$
\tilde{b}_{B L}=0.00005, \quad \tilde{a}_{B L}=0.03278, \quad \tilde{k}_{B L}=-0.40271
$$

Also under the Stein loss function, the Bayes estimate of the parameters $b$ and $a$ using (3.30) are given as

$$
\tilde{b}_{B S T}=0.00003, \quad \tilde{a}_{B S T}=0.02686 .
$$

Furthermore, from (4.5) the 95\% HPD credible interval of the parameters $b, a$ and $k$ are obtained as $\left(8.45 \times 10^{-6}, 1.03 \times 10^{-4}\right),(0.01071,0.06426)$ and $(-0.66441,-0.11864)$, respectively. 


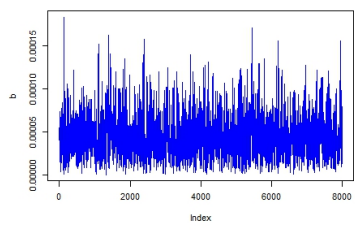

Histogram of $b$

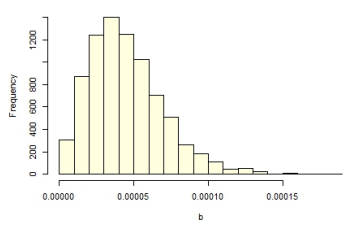

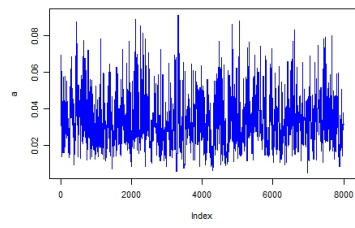

Histogram of a

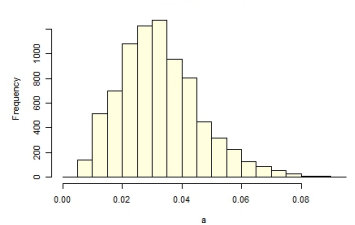

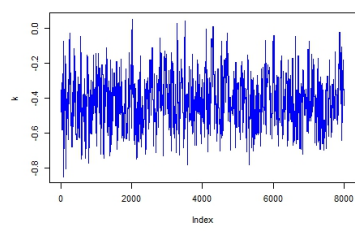

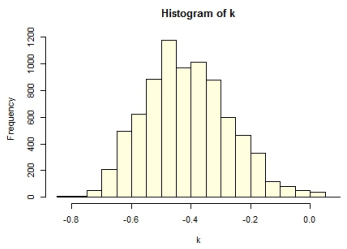

Figure 3. Simulated values and Histogram of the parameters $b, a$ and $k$

\section{A simulation study}

In this section, a simulation study is conducted to investigate and compare the performance of the presented estimators in the previous sections. We compare the performance of the MLEs using the EM and NR algorithms and Bayes estimators in terms of their estimated risk (ER). The performances of the confidence and credible intervals are also compared in terms of their coverage probability and expected length. When $\theta$ is estimated by $\hat{\theta}$, the ER of $\theta$ under the SEL function is given by

$$
E R_{B S}(\theta)=\frac{1}{T} \sum_{i=1}^{T}\left(\hat{\theta}_{i}-\theta\right)^{2}
$$

where $T$ is the number of replications and $\hat{\theta}_{i}$ is the estimate of $\theta$ in $i$ th replication. Moreover, the ER of $\theta$ under the LINEX and Stein loss functions are given by

$$
\begin{gathered}
E R_{B L}(\theta)=\frac{1}{T} \sum_{i=1}^{T}\left(e^{c\left(\hat{\theta}_{i}-\theta\right)}-c\left(\hat{\theta}_{i}-\theta\right)-1\right), \\
E R_{B S T}(\theta)=\frac{1}{T} \sum_{i=1}^{T}\left(\frac{\hat{\theta}_{i}}{\theta}-\ln \frac{\hat{\theta}_{i}}{\theta}-1\right),
\end{gathered}
$$

respectively. We generate progressively type-II censored sample of size $m$ from a sample of size $n$ drawn from P-LHRD using the algorithm suggested in Subsection 4.2. For each value of $n=20,50$, we consider three values of $m$ with different censoring schemes. Note that, for example, $\left(0^{* 4}\right)$ denotes $(0,0,0,0)$. We use two different values for the parameters $(b, a, k)$, namely $(1,0.5,-0.25)$ and $(0.25,0.5,0.1)$, respectively. To compute different Bayes estimates, we use the small hyperparameter values as $\alpha_{i}=1.5, \eta_{i}=\gamma_{i}=0.5$, $i=1,2,3$. We generate $N=10000$ MCMC samples and discard the first $M=2000$ values as burn-in period as described in Subsection 3.2. We report the average estimates and ER of the parameters in Tables 3-6. The expected length and coverage probability of the confidence and credible intervals for confidence level $(1-\alpha)=0.95$ are also reported in Tables 7 and 8. To compute the bootstrap confidence intervals, we use 500 bootstrap iterations. All the results are reported based on 1000 replications. 
Table 3. Average estimates and estimated risk (in parentheses) of the parameters for values of $(b=1, a=0.5, k=-0.25)$ with $n=20$.

\begin{tabular}{|c|c|c|c|c|c|c|c|c|}
\hline$m$ & Scheme & $\theta$ & $\hat{\theta}_{E M}$ & $\hat{\theta}_{N R}$ & $\tilde{\theta}_{B S}$ & $\begin{array}{c}\tilde{\theta}_{B L} \\
(c=2)\end{array}$ & $\begin{array}{c}\tilde{\theta}_{B L} \\
(c=-1)\end{array}$ & $\tilde{\theta}_{B S T}$ \\
\hline \multirow{18}{*}{10} & \multirow{6}{*}{$\left(10,0^{* 9}\right)$} & \multirow[t]{2}{*}{$b$} & 1.2802 & 1.2061 & 1.2739 & 0.8579 & 1.3118 & 0.6112 \\
\hline & & & $(0.6798)$ & $(0.8006)$ & $(0.3667)$ & $(0.3393)$ & $(0.2961)$ & $(0.2653)$ \\
\hline & & \multirow[t]{2}{*}{$a$} & 0.5781 & 0.6610 & 0.9558 & 0.7027 & 1.0394 & 0.6668 \\
\hline & & & $(0.2334)$ & $(0.4618)$ & $(0.3512)$ & $(0.3847)$ & $(0.3997)$ & \multirow[t]{3}{*}{$(0.2178)$} \\
\hline & & \multirow[t]{2}{*}{$k$} & -0.2116 & -0.1528 & 0.1559 & 0.0514 & 0.1423 & \\
\hline & & & $(0.0726)$ & $(0.1487)$ & $(0.4161)$ & $(0.5969)$ & $(0.6182)$ & \\
\hline & \multirow{8}{*}{$\left(0^{* 9}, 10\right)$} & \multirow[t]{2}{*}{$b$} & 1.3663 & 1.6984 & 0.6867 & 0.5862 & 1.3056 & 1.3774 \\
\hline & & & $(0.8287)$ & $(1.8454)$ & $(0.8650)$ & $(0.9943)$ & $(0.4355)$ & $(0.4445)$ \\
\hline & & \multirow[t]{2}{*}{$a$} & 0.4172 & 0.5989 & 0.8928 & 0.8827 & 0.8036 & \multirow{4}{*}{$\begin{array}{c}0.8400 \\
(0.5536)\end{array}$} \\
\hline & & & $(0.1827)$ & $(0.5528)$ & $(0.7119)$ & $(0.7480)$ & $(0.4799)$ & \\
\hline & & \multirow[t]{2}{*}{$k$} & -0.3571 & -0.1336 & 0.0771 & 0.1125 & 0.0979 & \\
\hline & & & $(0.0915)$ & $(0.1850)$ & $(0.3054)$ & $(0.7029)$ & $(0.3542)$ & \\
\hline & & \multirow[t]{2}{*}{$b$} & 1.3476 & 1.5485 & 1.4174 & 0.8245 & 1.4945 & 0.7110 \\
\hline & & & $(0.7093)$ & (1.5313) & $(0.5432)$ & $(0.6143)$ & $(0.4271)$ & $(0.4276)$ \\
\hline & \multirow[t]{4}{*}{$\left(1^{* 10}\right)$} & \multirow[t]{2}{*}{$a$} & 0.5639 & 0.6282 & 1.0411 & 0.8419 & 0.9012 & \multirow{4}{*}{$\begin{array}{c}0.6980 \\
(0.3594)\end{array}$} \\
\hline & & & $(0.1987)$ & $(0.5211)$ & $(0.4449)$ & $(0.5146)$ & $(0.3727)$ & \\
\hline & & \multirow[t]{2}{*}{$k$} & -0.3454 & -0.1202 & 0.1042 & 0.0983 & 0.1183 & \\
\hline & & & $(0.0824)$ & $(0.1572)$ & $(0.3124)$ & $(0.3968)$ & $(0.3865)$ & \\
\hline & & $b$ & 1.2774 & 1.1992 & 1.1445 & 0.7987 & 1.3844 & 0.6506 \\
\hline & & & $(0.5596)$ & $(0.7004)$ & $(0.2792)$ & $(0.2225)$ & $(0.2023)$ & $(0.2180)$ \\
\hline & $\left(8,0^{* 11}\right)$ & $a$ & 0.5529 & 0.6402 & 0.9473 & 0.7076 & 1.0483 & 0.5918 \\
\hline & & & $(0.2110)$ & $(0.3633)$ & $(0.3263)$ & $(0.3485)$ & $(0.3193)$ & $(0.1577)$ \\
\hline & & $k$ & -0.2219 & -0.1681 & 0.1093 & 0.0609 & 0.1264 & \\
\hline & & & $(0.0609)$ & $(0.1322)$ & $(0.3202)$ & $(0.4770)$ & $(0.4953)$ & \\
\hline & & $b$ & 1.3377 & 1.4832 & 1.5193 & 1.1204 & 1.3663 & 1.2294 \\
\hline & & & $(0.7502)$ & $(1.2551)$ & $(0.7687)$ & $(0.9785)$ & $(0.3919)$ & $(0.3854)$ \\
\hline 12 & $\left(0^{* 11}, 8\right)$ & $a$ & 0.4259 & 0.6064 & 0.9867 & 0.9706 & 0.8103 & 0.8999 \\
\hline & & & $(0.1618)$ & $(0.4606)$ & $(0.6287)$ & $(0.6810)$ & $(0.3836)$ & $(0.4976)$ \\
\hline & & $k$ & -0.3338 & -0.1416 & 0.0474 & 0.0186 & 0.0524 & \\
\hline & & & $(0.0818)$ & $(0.1784)$ & $(0.2510)$ & $(0.6096)$ & $(0.2956)$ & \\
\hline & & $b$ & 01.3430 & 1.3784 & 1.2384 & 0.8438 & 1.3343 & 0.7028 \\
\hline & & & $(0.6476)$ & $(1.1239)$ & $(0.4087)$ & $(0.4616)$ & $(0.3273)$ & $(0.3520)$ \\
\hline & $\left(0^{* 2}, 1^{* 8}, 0^{* 2}\right)$ & $a$ & 0.5474 & 0.6301 & 0.9352 & 0.8043 & 1.0603 & 0.6806 \\
\hline & & & $(0.1865)$ & $(0.4127)$ & $(0.3405)$ & $(0.3847)$ & $(0.2995)$ & $(0.2444)$ \\
\hline & & $k$ & -0.2713 & -0.1522 & 0.0313 & -0.0181 & 0.0527 & \\
\hline & & & $(0.0700)$ & $(0.1440)$ & $(0.2118)$ & $(0.3399)$ & $(0.3012)$ & \\
\hline & & $b$ & 1.1501 & 1.1469 & 1.0655 & 0.7787 & 1.2941 & 0.6481 \\
\hline & & & $(0.4085)$ & $(0.5172)$ & $(0.2678)$ & $(0.1964)$ & $(0.1628)$ & $(0.1899)$ \\
\hline & $\left(5,0^{* 14}\right)$ & $a$ & 0.5438 & 0.6044 & 0.8841 & 0.6824 & 1.1013 & 0.5647 \\
\hline & & & $(0.1818)$ & $(0.3053)$ & $(0.2529)$ & $(0.2581)$ & $(0.2277)$ & $(0.1381)$ \\
\hline & & $k$ & -0.2309 & -0.1875 & 0.0858 & -0.0421 & 0.0603 & \\
\hline & & & $(0.0549)$ & $(0.1102)$ & $(0.2875)$ & $(0.3741)$ & $(0.4019)$ & \\
\hline & & $b$ & 1.2945 & 1.3106 & 1.6044 & 1.2801 & 1.3843 & 1.1282 \\
\hline & & & $(0.6656)$ & $(0.8236)$ & $(0.6162)$ & $(0.8462)$ & $(0.3165)$ & $(0.3344)$ \\
\hline 15 & $\left(0^{* 14}, 5\right)$ & $a$ & 0.4722 & 0.5973 & 1.0517 & 0.8964 & 0.7104 & 0.8684 \\
\hline & & & $(0.1408)$ & $(0.4127)$ & $(0.5738)$ & $(0.6163)$ & $(0.2573)$ & $(0.4227)$ \\
\hline & & $k$ & -0.2717 & -0.1788 & 0.0846 & 0.0895 & 0.0804 & \\
\hline & & & $(0.0664)$ & $(0.1501)$ & $(0.1679)$ & $(0.5188)$ & $(0.2563)$ & \\
\hline & & $b$ & 1.2601 & 1.1328 & 1.2983 & 0.7627 & 1.4351 & 0.6188 \\
\hline & & & $(0.5585)$ & $(0.7085)$ & $(0.3681)$ & $(0.3727)$ & $(0.2594)$ & $(0.2756)$ \\
\hline & $\left(0^{* 5}, 1^{* 5}, 0^{* 5}\right)$ & $a$ & 0.5229 & 0.7004 & 0.9182 & 0.9043 & 1.0435 & 0.6026 \\
\hline & & & $(0.1539)$ & $(0.3853)$ & $(0.2786)$ & $(0.3226)$ & $(0.2458)$ & $(0.1993)$ \\
\hline & & $k$ & -0.2858 & -0.1458 & -0.0116 & -0.0401 & 0.0877 & \\
\hline & & & $(0.0595)$ & $(0.1248)$ & $(0.1986)$ & $(0.2427)$ & $(0.2224)$ & \\
\hline
\end{tabular}


Table 4. Average estimates and estimated risk (in parentheses) of the parameters for values of $(b=1, a=0.5, k=-0.25)$ with $n=50$.

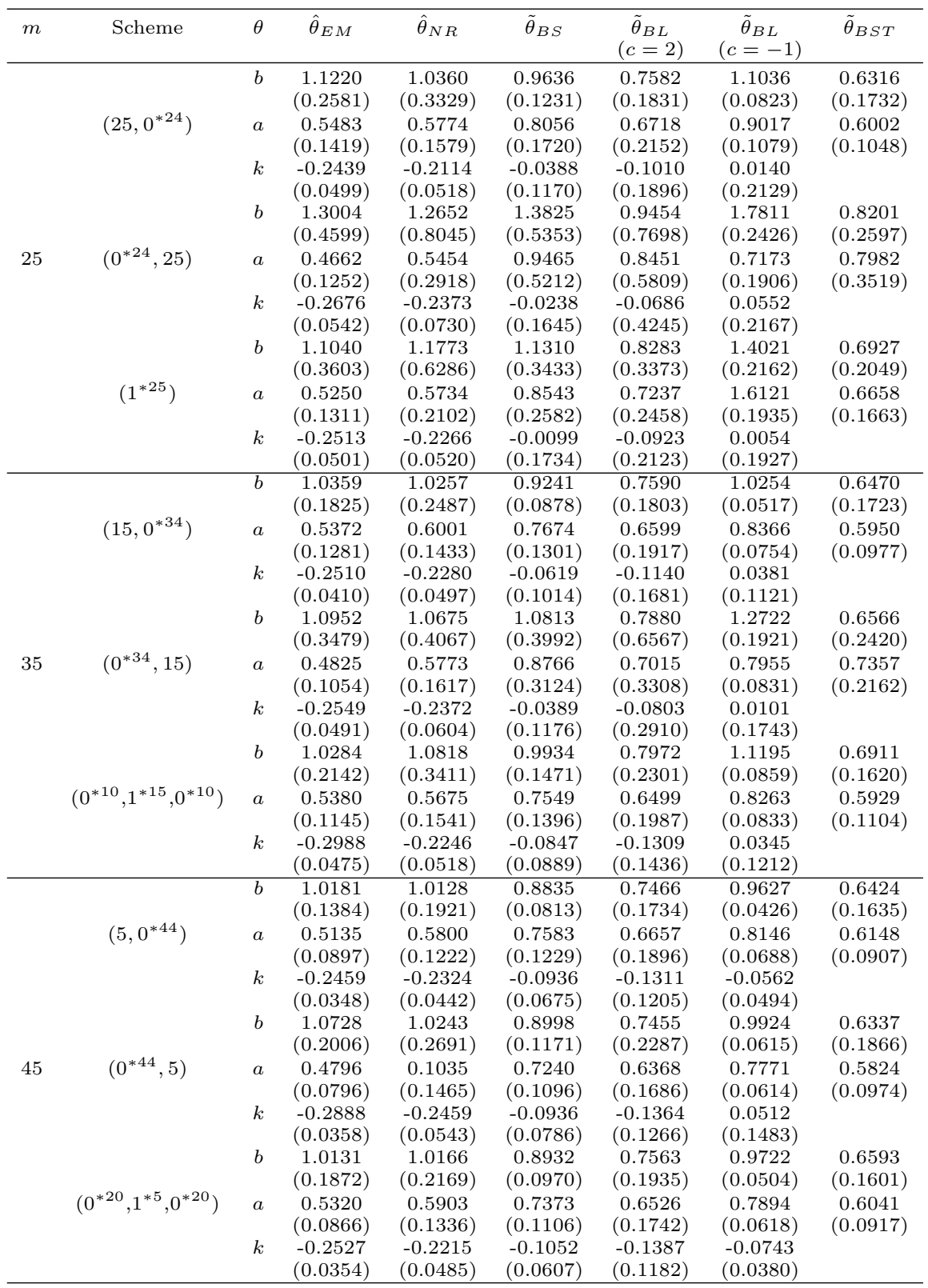


Table 5. Average estimates and estimated risk (in parentheses) of the parameters for values of $(b=0.25, a=0.5, k=0.1)$ with $n=20$.

\begin{tabular}{|c|c|c|c|c|c|c|c|c|}
\hline$m$ & Scheme & $\theta$ & $\hat{\theta}_{E M}$ & $\hat{\theta}_{N R}$ & $\tilde{\theta}_{B S}$ & $\begin{array}{c}\tilde{\theta}_{B L} \\
(c=2)\end{array}$ & $\begin{array}{c}\tilde{\theta}_{B L} \\
(c=-1)\end{array}$ & $\tilde{\theta}_{B S T}$ \\
\hline \multirow{9}{*}{10} & \multirow{3}{*}{$\left(10,0^{* 9}\right)$} & $b$ & $\begin{array}{c}0.4247 \\
(0.1408)\end{array}$ & $\begin{array}{c}0.3065 \\
(0.1766)\end{array}$ & $\begin{array}{c}0.5360 \\
(0.1536)\end{array}$ & $\begin{array}{c}0.4488 \\
(0.2136)\end{array}$ & $\begin{array}{c}0.5973 \\
(0.0886)\end{array}$ & $\begin{array}{c}0.3305 \\
(0.1479)\end{array}$ \\
\hline & & $a$ & $\begin{array}{c}0.3703 \\
(0.0763)\end{array}$ & $\begin{array}{c}0.6164 \\
(0.2046)\end{array}$ & $\begin{array}{c}0.5418 \\
(0.0483)\end{array}$ & $\begin{array}{c}0.4379 \\
(0.0511)\end{array}$ & $\begin{array}{c}0.5873 \\
(0.0346)\end{array}$ & $\begin{array}{c}0.3184 \\
(0.1669)\end{array}$ \\
\hline & & $k$ & $\begin{array}{l}-0.0545 \\
(0.1292)\end{array}$ & $\begin{array}{c}0.2877 \\
(0.3131)\end{array}$ & $\begin{array}{c}0.4662 \\
(0.3680)\end{array}$ & $\begin{array}{c}0.2541 \\
(0.3854)\end{array}$ & $\begin{array}{c}0.8091 \\
(0.3327)\end{array}$ & \\
\hline & \multirow{4}{*}{$\left(0^{* 9}, 10\right)$} & $b$ & $\begin{array}{c}0.4456 \\
(0.2033)\end{array}$ & $\begin{array}{c}0.4216 \\
(0.2868)\end{array}$ & $\begin{array}{c}0.7140 \\
(0.3984)\end{array}$ & $\begin{array}{c}0.6581 \\
(0.6484)\end{array}$ & $\begin{array}{c}0.7420 \\
(0.1977)\end{array}$ & $\begin{array}{c}0.4692 \\
(0.4020)\end{array}$ \\
\hline & & $a$ & $\begin{array}{c}0.3512 \\
(0.1110)\end{array}$ & $\begin{array}{c}0.7110 \\
(0.4313)\end{array}$ & $\begin{array}{c}0.5668 \\
(0.0915)\end{array}$ & $\begin{array}{c}0.4259 \\
(0.3115)\end{array}$ & $\begin{array}{c}0.6423 \\
(0.1599)\end{array}$ & $\begin{array}{c}0.3118 \\
(0.3093)\end{array}$ \\
\hline & & $k$ & $\begin{array}{l}-0.0826 \\
(0.1572)\end{array}$ & $\begin{array}{c}0.3776 \\
(0.3837)\end{array}$ & $\begin{array}{c}0.6425 \\
(0.5854)\end{array}$ & $\begin{array}{c}0.3823 \\
(0.4879)\end{array}$ & $\begin{array}{c}0.9674 \\
(0.5383)\end{array}$ & \\
\hline & & $b$ & $\begin{array}{c}0.4596 \\
(0.1869)\end{array}$ & $\begin{array}{c}0.3201 \\
(0.2166)\end{array}$ & $\begin{array}{c}0.6499 \\
(0.2777)\end{array}$ & $\begin{array}{c}0.5277 \\
(0.4485)\end{array}$ & $\begin{array}{c}0.7381 \\
(0.1512)\end{array}$ & $\begin{array}{c}0.3992 \\
(0.2789)\end{array}$ \\
\hline & \multirow[t]{2}{*}{$\left(1^{* 10}\right)$} & $a$ & $\begin{array}{c}0.3623 \\
(0.0944)\end{array}$ & $\begin{array}{c}0.7203 \\
(0.3790)\end{array}$ & $\begin{array}{c}0.5441 \\
(0.0558)\end{array}$ & $\begin{array}{c}0.4565 \\
(0.1134)\end{array}$ & $\begin{array}{c}0.6184 \\
(0.0365)\end{array}$ & $\begin{array}{c}0.3282 \\
(0.1948)\end{array}$ \\
\hline & & $k$ & $\begin{array}{l}-0.0811 \\
(0.1479)\end{array}$ & $\begin{array}{c}0.3686 \\
(0.3495)\end{array}$ & $\begin{array}{c}0.6399 \\
(0.5532)\end{array}$ & $\begin{array}{c}0.3202 \\
(0.4641)\end{array}$ & $\begin{array}{c}0.9328 \\
(0.4503)\end{array}$ & \\
\hline \multirow{9}{*}{12} & \multirow{3}{*}{$\left(8,0^{* 11}\right)$} & $b$ & $\begin{array}{c}0.4054 \\
(0.1179)\end{array}$ & $\begin{array}{c}0.2845 \\
(0.1283)\end{array}$ & $\begin{array}{c}0.4956 \\
(0.1100)\end{array}$ & $\begin{array}{c}0.4246 \\
(0.1543)\end{array}$ & $\begin{array}{c}0.5415 \\
(0.0627)\end{array}$ & $\begin{array}{c}0.3091 \\
(0.1056)\end{array}$ \\
\hline & & $a$ & $\begin{array}{c}0.3868 \\
(0.0681)\end{array}$ & $\begin{array}{c}0.6158 \\
(0.1588)\end{array}$ & $\begin{array}{c}0.5377 \\
(0.0433)\end{array}$ & $\begin{array}{c}0.4584 \\
(0.0481)\end{array}$ & $\begin{array}{c}0.5908 \\
(0.0302)\end{array}$ & $\begin{array}{c}0.3067 \\
(0.1608)\end{array}$ \\
\hline & & $k$ & $\begin{array}{l}-0.0308 \\
(0.1107)\end{array}$ & $\begin{array}{c}0.2819 \\
(0.2724)\end{array}$ & $\begin{array}{c}0.4167 \\
(0.2916)\end{array}$ & $\begin{array}{c}0.2655 \\
(0.3292)\end{array}$ & $\begin{array}{c}0.5597 \\
(0.2437)\end{array}$ & \\
\hline & \multirow{6}{*}{$\left(0^{* 11}, 8\right)$} & $b$ & $\begin{array}{c}0.4241 \\
(0.1641)\end{array}$ & $\begin{array}{c}0.3550 \\
(0.1817)\end{array}$ & $\begin{array}{c}0.6390 \\
(0.2371)\end{array}$ & $\begin{array}{c}0.5250 \\
(0.5034)\end{array}$ & $\begin{array}{c}0.7662 \\
(0.1452)\end{array}$ & $\begin{array}{c}0.4188 \\
(0.3320)\end{array}$ \\
\hline & & $a$ & $\begin{array}{c}0.3667 \\
(0.0997)\end{array}$ & $\begin{array}{c}0.6508 \\
(0.2653)\end{array}$ & $\begin{array}{c}0.5441 \\
(0.0757)\end{array}$ & $\begin{array}{c}0.4619 \\
(0.2925)\end{array}$ & $\begin{array}{c}0.6805 \\
(0.0912)\end{array}$ & $\begin{array}{c}0.3365 \\
(0.2685)\end{array}$ \\
\hline & & $k$ & $\begin{array}{l}-0.0772 \\
(0.1435)\end{array}$ & $\begin{array}{c}0.3675 \\
(0.3654)\end{array}$ & $\begin{array}{c}0.5821 \\
(0.3805)\end{array}$ & $\begin{array}{c}0.3218 \\
(0.3012)\end{array}$ & $\begin{array}{c}0.4354 \\
(0.4736)\end{array}$ & \\
\hline & & $b$ & $\begin{array}{c}0.4327 \\
(0.1345)\end{array}$ & $\begin{array}{c}0.2801 \\
(0.1406)\end{array}$ & $\begin{array}{c}0.5590 \\
(0.1722)\end{array}$ & $\begin{array}{c}0.4695 \\
(0.2842)\end{array}$ & $\begin{array}{c}0.6175 \\
(0.0916)\end{array}$ & $\begin{array}{c}0.3430 \\
(0.1529)\end{array}$ \\
\hline & & $a$ & $\begin{array}{c}0.3606 \\
(0.0761)\end{array}$ & $\begin{array}{c}0.6531 \\
(0.2464)\end{array}$ & $\begin{array}{c}0.5468 \\
(0.0521)\end{array}$ & $\begin{array}{c}0.4289 \\
(0.0828)\end{array}$ & $\begin{array}{c}0.6116 \\
(0.0355)\end{array}$ & $\begin{array}{c}0.3164 \\
(0.1835)\end{array}$ \\
\hline & & $k$ & $\begin{array}{c}-0.0777 \\
(0.1318)\end{array}$ & $\begin{array}{c}0.3025 \\
(0.2940)\end{array}$ & $\begin{array}{c}0.5038 \\
(0.3822)\end{array}$ & $\begin{array}{c}0.2761 \\
(0.3420)\end{array}$ & $\begin{array}{c}0.8986 \\
(0.3992)\end{array}$ & \\
\hline \multirow{9}{*}{15} & \multirow{3}{*}{$\left(5,0^{* 14}\right)$} & $b$ & $\begin{array}{c}0.3813 \\
(0.0880)\end{array}$ & $\begin{array}{c}0.2261 \\
(0.0945)\end{array}$ & $\begin{array}{c}0.4541 \\
(0.0826)\end{array}$ & $\begin{array}{c}0.3968 \\
(0.1180)\end{array}$ & $\begin{array}{c}0.4894 \\
(0.0468)\end{array}$ & $\begin{array}{c}0.2898 \\
(0.1017)\end{array}$ \\
\hline & & $a$ & $\begin{array}{c}0.4177 \\
(0.0591)\end{array}$ & $\begin{array}{c}0.5991 \\
(0.1405)\end{array}$ & $\begin{array}{c}0.4825 \\
(0.0240)\end{array}$ & $\begin{array}{c}0.4217 \\
(0.0370)\end{array}$ & $\begin{array}{c}0.5216 \\
(0.0160)\end{array}$ & $\begin{array}{c}0.3176 \\
(0.1546)\end{array}$ \\
\hline & & $k$ & $\begin{array}{l}-0.0115 \\
(0.0996)\end{array}$ & $\begin{array}{c}0.2789 \\
(0.2689)\end{array}$ & $\begin{array}{c}0.4238 \\
(0.2423)\end{array}$ & $\begin{array}{c}0.2276 \\
(0.2645)\end{array}$ & $\begin{array}{c}0.6023 \\
(0.2033)\end{array}$ & \\
\hline & \multirow{3}{*}{$\left(0^{* 14}, 5\right)$} & $b$ & $\begin{array}{c}0.4112 \\
(0.1387)\end{array}$ & $\begin{array}{c}0.3149 \\
(0.1431)\end{array}$ & $\begin{array}{c}0.5512 \\
(0.1842)\end{array}$ & $\begin{array}{c}0.4652 \\
(0.4067)\end{array}$ & $\begin{array}{c}0.6051 \\
(0.0997)\end{array}$ & $\begin{array}{c}0.3329 \\
(0.3175)\end{array}$ \\
\hline & & $a$ & $\begin{array}{c}0.3829 \\
(0.0890)\end{array}$ & $\begin{array}{c}0.6093 \\
(0.1683)\end{array}$ & $\begin{array}{c}0.4822 \\
(0.0366)\end{array}$ & $\begin{array}{c}0.4249 \\
(0.0888)\end{array}$ & $\begin{array}{c}0.5468 \\
(0.0213)\end{array}$ & $\begin{array}{c}0.2957 \\
(0.1848)\end{array}$ \\
\hline & & $k$ & $\begin{array}{c}-0.0308 \\
(0.1284)\end{array}$ & $\begin{array}{c}0.3313 \\
(0.3319)\end{array}$ & $\begin{array}{c}0.4392 \\
(0.3224)\end{array}$ & $\begin{array}{c}0.1945 \\
(0.2960)\end{array}$ & $\begin{array}{c}0.7645 \\
(0.3893)\end{array}$ & \\
\hline & \multirow{3}{*}{$\left(0^{* 5}, 1^{* 5}, 0^{* 5}\right)$} & $b$ & $\begin{array}{c}0.4248 \\
(0.1063)\end{array}$ & $\begin{array}{c}0.2498 \\
(0.1204)\end{array}$ & $\begin{array}{c}0.5068 \\
(0.1222)\end{array}$ & $\begin{array}{c}0.4365 \\
(0.1856)\end{array}$ & $\begin{array}{c}0.5507 \\
(0.0673)\end{array}$ & $\begin{array}{c}0.3249 \\
(0.1442)\end{array}$ \\
\hline & & $a$ & $\begin{array}{c}0.3751 \\
(0.0674)\end{array}$ & $\begin{array}{c}0.6283 \\
(0.1595)\end{array}$ & $\begin{array}{c}0.4980 \\
(0.0313)\end{array}$ & $\begin{array}{c}0.4349 \\
(0.0757)\end{array}$ & $\begin{array}{c}0.5383 \\
(0.0184)\end{array}$ & $\begin{array}{c}0.3267 \\
(0.1561)\end{array}$ \\
\hline & & $k$ & $\begin{array}{c}-0.0696 \\
(0.1145)\end{array}$ & $\begin{array}{c}0.3180 \\
(0.2759)\end{array}$ & $\begin{array}{c}0.4022 \\
(0.3017)\end{array}$ & $\begin{array}{c}0.2022 \\
(0.2904)\end{array}$ & $\begin{array}{c}0.7462 \\
(0.3357)\end{array}$ & \\
\hline
\end{tabular}


Table 6. Average estimates and estimated risk (in parentheses) of the parameters for values of $(b=0.25, a=0.5, k=0.1)$ with $n=50$.

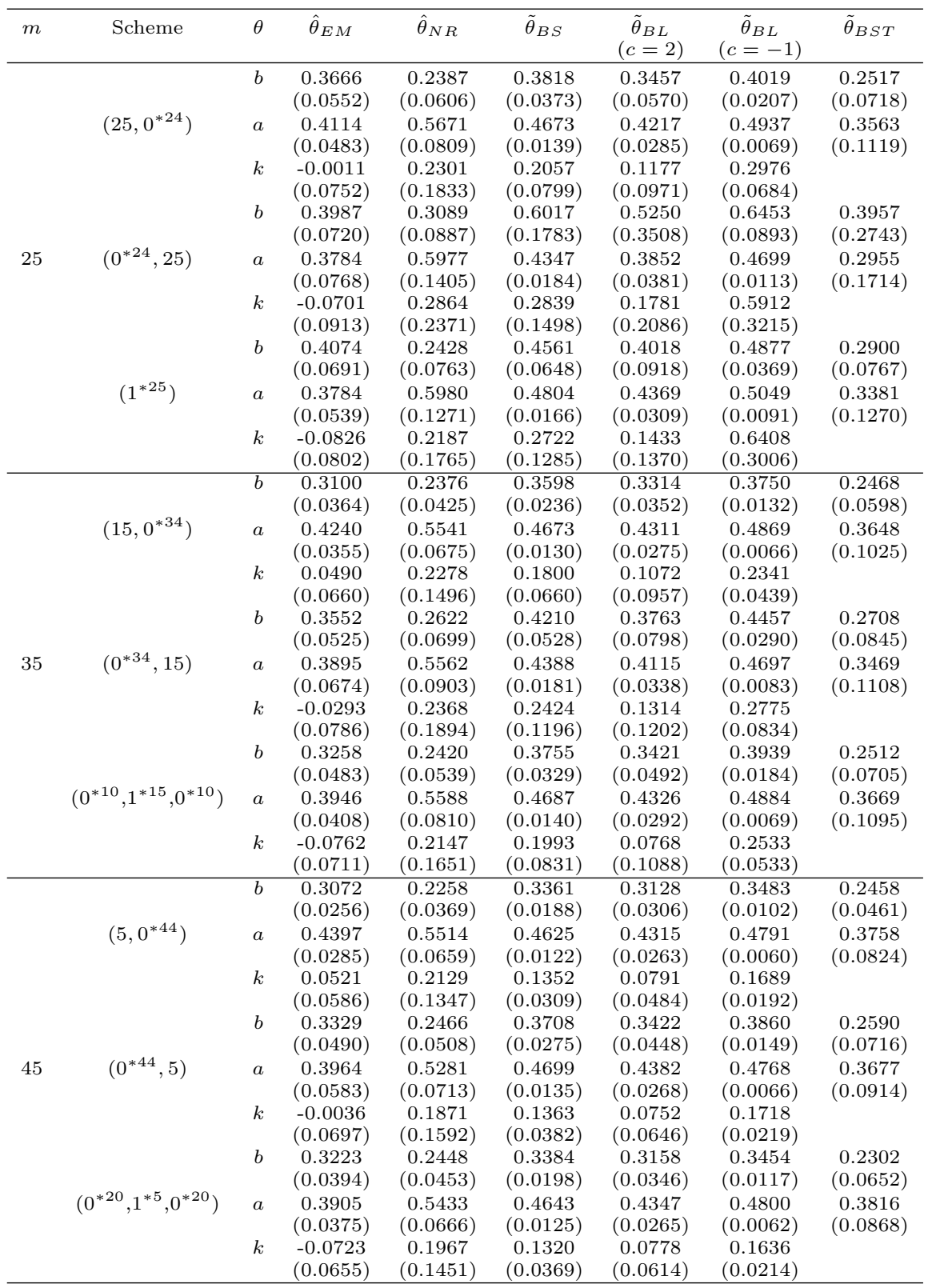


Table 7. Expected lengths and coverage probabilities (in parentheses) of the parameters for values of $(b=1, a=0.5, k=-0.25)$.

\begin{tabular}{|c|c|c|c|c|c|}
\hline$(n, m)$ & Scheme & Methods & $b$ & $a$ & $k$ \\
\hline \multirow{13}{*}{$(20,10)$} & $\left(10,0^{* 9}\right)$ & AMLE & $3.4958(0.926)$ & $2.7145(0.868)$ & $1.6283(0.872)$ \\
\hline & & Boot-p & $2.3552(0.986)$ & $1.6946(0.979)$ & $1.2693(0.970)$ \\
\hline & & Boot-t & $2.7587(0.981)$ & $1.9182(0.963)$ & $2.8534(0.954)$ \\
\hline & & MCMC & $2.7733(0.972)$ & $2.2483(0.968)$ & $1.9145(0.884)$ \\
\hline & $\left(0^{* 9}, 10\right)$ & AMLE & $5.1430(0.929)$ & $2.9574(0.847)$ & $1.5228(0.839)$ \\
\hline & & Boot-p & $2.4491(0.982)$ & $1.7015(0.970)$ & $1.1113(0.964)$ \\
\hline & & Boot-t & $2.9148(0.969)$ & $1.9243(0.966)$ & $2.5006(0.953)$ \\
\hline & & MCMC & $2.5423(0.959)$ & $2.8899(0.949)$ & $2.1130(0.880)$ \\
\hline & $(1 * 10)$ & AMLE & $4.6610(0.941)$ & $2.8399(0.856)$ & $1.5652(0.853)$ \\
\hline & & Boot-p & $2.4777(0.987)$ & $1.7099(0.983)$ & $1.1045(0.958)$ \\
\hline & & Boot-t & $2.6734(0.975)$ & $1.8833(0.961)$ & $2.0569(0.955)$ \\
\hline & & MCMC & $2.7704(0.964)$ & $2.5157(0.955)$ & $2.0374(0.883)$ \\
\hline & $\left(8,0^{* 11}\right)$ & AMLE & $3.3371(0.936)$ & $2.5627(0.866)$ & $1.5484(0.846)$ \\
\hline \multirow{7}{*}{$(20,12)$} & & Boot-p & $2.3097(0.981)$ & $1.5445(0.949)$ & $1.0972(0.954)$ \\
\hline & & Boot-t & $2.7483(0.985)$ & $1.6167(0.943)$ & $2.3354(0.961)$ \\
\hline & & MCMC & $2.4922(0.975)$ & $2.1043(0.965)$ & $1.7921(0.883)$ \\
\hline & $\left(0^{* 11}, 8\right)$ & AMLE & $4.9543(0.933)$ & $2.8318(0.857)$ & $1.4512(0.833)$ \\
\hline & & Boot-p & $2.4463(0.989)$ & $1.6032(0.941)$ & $1.0596(0.945)$ \\
\hline & & Boot-t & $2.7134(0.978)$ & $1.7057(0.950)$ & $2.1490(0.968)$ \\
\hline & & MCMC & $2.7708(0.953)$ & $2.7218(0.947)$ & $1.9228(0.876)$ \\
\hline \multirow{16}{*}{$(20,15)$} & $\left(0^{* 2}, 1^{* 8}, 0^{* 2}\right)$ & AMLE & $3.6079(0.925)$ & $2.6241(0.848)$ & $1.4062(0.849)$ \\
\hline & & Boot-p & $2.4399(0.982)$ & $1.6404(0.951)$ & $1.0599(0.951)$ \\
\hline & & Boot-t & $2.6109(0.990)$ & $1.6851(0.955)$ & $1.9091(0.957)$ \\
\hline & & MCMC & $2.6784(0.958)$ & $2.3982(0.961)$ & $1.8374(0.875)$ \\
\hline & $\left(5,0^{* 14}\right)$ & AMLE & $3.0648(0.931)$ & $2.4497(0.878)$ & $1.4578(0.868)$ \\
\hline & & Boot-p & $2.2626(0.977)$ & $1.4745(0.960)$ & $1.0763(0.951)$ \\
\hline & & Boot-t & $2.6690(0.973)$ & $1.5950(0.949)$ & $1.5504(0.947)$ \\
\hline & & MCMC & $2.2858(0.975)$ & $1.7547(0.969)$ & $1.5034(0.865)$ \\
\hline & $(0 * 14,5)$ & AMLE & $4.7238(0.934)$ & $2.7064(0.874)$ & $1.4186(0.831)$ \\
\hline & & Boot-p & $2.3815(0.981)$ & $1.5996(0.958)$ & $1.0413(0.945)$ \\
\hline & & Boot-t & $2.6676(0.984)$ & $1.6504(0.955)$ & $1.5612(0.953)$ \\
\hline & & MCMC & $2.9359(0.969)$ & $1.9693(0.955)$ & $1.7213(0.860)$ \\
\hline & $\left(0^{* 5}, 1^{* 5}, 0^{* 5}\right)$ & AMLE & $3.5573(0.926)$ & $2.5570(0.876)$ & $1.3951(0.857)$ \\
\hline & & Boot-p & $2.3353(0.982)$ & $1.5753(0.959)$ & $1.0188(0.954)$ \\
\hline & & Boot-t & $2.5549(0.985)$ & $1.6549(0.948)$ & $1.4686(0.941)$ \\
\hline & & MCMC & $2.7883(0.973)$ & $1.8635(0.960)$ & $1.6656(0.858)$ \\
\hline \multirow{12}{*}{$(50,25)$} & $\left(25,0^{* 24}\right)$ & AMLE & $2.5966(0.928)$ & $2.0389(0.895)$ & $1.1536(0.904)$ \\
\hline & & Boot-p & $2.0606(0.979)$ & $1.4611(0.961)$ & $0.8981(0.952)$ \\
\hline & & Boot-t & $2.4521(0.967)$ & $1.5423(0.957)$ & $0.9983(0.938)$ \\
\hline & & MCMC & $1.8072(0.982)$ & $1.4684(0.958)$ & $0.9876(0.862)$ \\
\hline & $\left(0^{* 24}, 25\right)$ & AMLE & $4.2745(0.930)$ & $2.1567(0.904)$ & $1.1522(0.864)$ \\
\hline & & Boot-p & $2.2814(0.971)$ & $1.5799(0.959)$ & $0.9981(0.958)$ \\
\hline & & Boot-t & $2.6548(0.973)$ & $1.6257(0.955)$ & $1.1306(0.942)$ \\
\hline & & $\mathrm{MCMC}$ & $2.7038(0.959)$ & $1.9323(0.935)$ & $1.0946(0.855)$ \\
\hline & $\left(1^{* 25}\right)$ & AMLE & $3.4424(0.927)$ & $1.9206(0.908)$ & $1.1005(0.897)$ \\
\hline & & Boot-p & $2.1813(0.968)$ & $1.5065(0.956)$ & $0.8986(0.949)$ \\
\hline & & Boot-t & $2.5146(0.975)$ & $1.6299(0.948)$ & $1.0352(0.945)$ \\
\hline & & MCMC & $2.3355(0.970)$ & $1.5544(0.940)$ & $1.0396(0.854)$ \\
\hline
\end{tabular}


Table 7: (Continued)

\begin{tabular}{|c|c|c|c|c|c|}
\hline$(n, m)$ & Scheme & Methods & $b$ & $a$ & $k$ \\
\hline \multirow{8}{*}{$(50,35)$} & \multirow{8}{*}{$\left(0^{* 34}, 15\right)$} & AMLE & $2.3368(0.943)$ & $1.9098(0.888)$ & $1.1319(0.932)$ \\
\hline & & Boot-p & $1.8475(0.988)$ & $1.3346(0.950)$ & $0.8348(0.963)$ \\
\hline & & Boot-t & $2.2181(0.980)$ & $1.4190(0.944)$ & $0.9539(0.952)$ \\
\hline & & MCMC & $1.5992(0.985)$ & $1.2869(0.943)$ & $0.8513(0.883)$ \\
\hline & & AMLE & $3.2584(0.924)$ & $1.8918(0.910)$ & $1.0821(0.886)$ \\
\hline & & Boot-p & $2.0374(0.972)$ & $1.3799(0.952)$ & $0.9628(0.955)$ \\
\hline & & Boot-t & $2.5873(0.976)$ & $1.5886(0.957)$ & $0.9773(0.946)$ \\
\hline & & MCMC & $2.0712(0.971)$ & $1.2474(0.927)$ & $0.8211(0.865)$ \\
\hline \multirow{16}{*}{$(50,45)$} & \multirow[t]{4}{*}{$\left(0^{* 10}, 1^{* 15}, 0^{* 10}\right)$} & AMLE & $2.4897(0.926)$ & $1.7900(0.897)$ & $1.0831(0.913)$ \\
\hline & & Boot-p & $2.0087(0.982)$ & $1.3245(0.947)$ & $0.8364(0.957)$ \\
\hline & & Boot-t & $2.3760(0.973)$ & $1.5771(0.946)$ & $0.9541(0.949)$ \\
\hline & & MCMC & $1.7441(0.976)$ & $1.2481(0.932)$ & $0.8855(0.876)$ \\
\hline & \multirow[t]{4}{*}{$\left(5,0^{* 44}\right)$} & AMLE & $2.1386(0.914)$ & $1.6701(0.902)$ & $1.1308(0.912)$ \\
\hline & & Boot-p & $1.7012(0.968)$ & $1.2542(0.953)$ & $0.7785(0.956)$ \\
\hline & & Boot-t & $1.9831(0.959)$ & $1.3102(0.945)$ & $0.8993(0.945)$ \\
\hline & & $\mathrm{MCMC}$ & $1.4441(0.973)$ & $1.1742(0.936)$ & $0.7143(0.869)$ \\
\hline & \multirow[t]{4}{*}{$\left(0^{* 44}, 5\right)$} & AMLE & $2.5908(0.932)$ & $1.6896(0.913)$ & $1.0309(0.904)$ \\
\hline & & Boot-p & $1.8332(0.975)$ & $1.2337(0.952)$ & $0.8321(0.941)$ \\
\hline & & Boot-t & $2.1141(0.966)$ & $1.2581(0.943)$ & $0.9529(0.946)$ \\
\hline & & MCMC & $1.5262(0.961)$ & $1.1254(0.915)$ & $0.7605(0.863)$ \\
\hline & \multirow[t]{4}{*}{$\left(0^{* 20}, 1^{* 5}, 0^{* 20}\right)$} & AMLE & $2.3220(0.925)$ & $1.6883(0.898)$ & $1.0487(0.923)$ \\
\hline & & Boot-p & $1.7418(0.970)$ & $1.2315(0.956)$ & $0.7994(0.950)$ \\
\hline & & Boot-t & $2.1021(0.972)$ & $1.2563(0.946)$ & $0.8740(0.942)$ \\
\hline & & MCMC & $1.4428(0.963)$ & $1.1215(0.923)$ & $0.6755(0.856)$ \\
\hline
\end{tabular}


Table 8. Expected lengths and coverage probabilities (in parentheses) of the parameters for values of $(b=0.25, a=0.5, k=0.1)$.

\begin{tabular}{|c|c|c|c|c|c|}
\hline$(n, m)$ & Scheme & Methods & $b$ & $a$ & $k$ \\
\hline \multirow{13}{*}{$(20,10)$} & \multirow[t]{4}{*}{$\left(10,0^{* 9}\right)$} & AMLE & $2.3295(0.947)$ & $2.9704(0.949)$ & $2.4451(0.907)$ \\
\hline & & Boot-p & $1.5279(0.991)$ & $1.5641(0.974)$ & $1.5534(0.953)$ \\
\hline & & Boot-t & $1.5650(0.983)$ & $1.6052(0.969)$ & $1.8805(0.951)$ \\
\hline & & MCMC & $1.1234(0.991)$ & $1.1250(0.993)$ & $2.2661(0.983)$ \\
\hline & \multirow[t]{4}{*}{$\left(0^{* 9}, 10\right)$} & AMLE & $3.5419(0.946)$ & $2.8077(0.940)$ & $2.0313(0.898)$ \\
\hline & & Boot-p & $1.8885(0.988)$ & $1.9394(0.971)$ & $1.5890(0.949)$ \\
\hline & & Boot-t & $1.9345(0.978)$ & $2.1384(0.973)$ & $2.2392(0.957)$ \\
\hline & & MCMC & $1.4378(0.985)$ & $1.3825(0.985)$ & $2.3953(0.969)$ \\
\hline & \multirow[t]{4}{*}{$\left(1^{* 10}\right)$} & AMLE & $2.5737(0.953)$ & $2.5831(0.947)$ & $2.2018(0.893)$ \\
\hline & & Boot-p & $1.7647(0.990)$ & $1.7385(0.977)$ & $1.5708(0.950)$ \\
\hline & & Boot-t & $1.7951(0.986)$ & $1.8061(0.970)$ & $1.9764(0.953)$ \\
\hline & & MCMC & $1.2506(0.987)$ & $1.2956(0.989)$ & $2.3235(0.875)$ \\
\hline & \multirow{4}{*}{$\left(8,0^{* 11}\right)$} & AMLE & $2.1086(0.941)$ & $2.6822(0.952)$ & $2.3754(0.894)$ \\
\hline \multirow{7}{*}{$(20,12)$} & & Boot-p & $1.4470(0.986)$ & $1.4512(0.980)$ & $1.5427(0.948)$ \\
\hline & & Boot-t & $1.4327(0.979)$ & $1.3773(0.971)$ & $1.7726(0.950)$ \\
\hline & & MCMC & $1.0033(0.996)$ & $1.0305(0.996)$ & $2.1933(0.988)$ \\
\hline & \multirow[t]{4}{*}{$\left(0^{* 11}, 8\right)$} & AMLE & $3.1709(0.954)$ & $2.7692(0.945)$ & $2.0200(0.879)$ \\
\hline & & Boot-p & $1.7612(0.992)$ & $1.6456(0.974)$ & $1.5779(0.955)$ \\
\hline & & Boot-t & $1.6996(0.984)$ & $1.6040(0.968)$ & $2.1397(0.953)$ \\
\hline & & MCMC & $1.2102(0.989)$ & $1.2828(0.988)$ & $2.3639(0.973)$ \\
\hline \multirow{16}{*}{$(20,15)$} & \multirow[t]{4}{*}{$\left(0^{* 2}, 1^{* 8}, 0^{* 2}\right)$} & AMLE & $2.2824(0.951)$ & $2.5678(0.954)$ & $2.1603(0.886)$ \\
\hline & & Boot-p & $1.6100(0.986)$ & $1.5582(0.979)$ & $1.5580(0.952)$ \\
\hline & & Boot-t & $1.6308(0.985)$ & $1.4686(0.972)$ & $1.9639(0.956)$ \\
\hline & & MCMC & $1.1455(0.988)$ & $1.1653(0.990)$ & $2.2121(0.977)$ \\
\hline & \multirow{4}{*}{$\left(5,0^{* 14}\right)$} & AMLE & $2.0439(0.959)$ & $2.6343(0.961)$ & $2.3463(0.904)$ \\
\hline & & Boot-p & $1.3242(0.993)$ & $1.3548(0.983)$ & $1.5298(0.958)$ \\
\hline & & Boot-t & $1.2636(0.980)$ & $1.1676(0.968)$ & $1.7526(0.955)$ \\
\hline & & MCMC & $0.8933(0.996)$ & $0.9459(0.996)$ & $1.9097(0.986)$ \\
\hline & \multirow[t]{4}{*}{$\left(0^{* 14}, 5\right)$} & AMLE & $2.4170(0.965)$ & $2.5157(0.960)$ & $2.0055(0.992)$ \\
\hline & & Boot-p & $1.6284(0.995)$ & $1.3985(0.976)$ & $1.5544(0.951)$ \\
\hline & & Boot-t & $1.4804(0.982)$ & $1.3571(0.967)$ & $1.9974(0.959)$ \\
\hline & & MCMC & $1.1065(0.991)$ & $1.0934(0.990)$ & $2.1149(0.979)$ \\
\hline & \multirow[t]{4}{*}{$\left(0^{* 5}, 1^{* 5}, 0^{* 5}\right)$} & AMLE & $2.2204(0.947)$ & $2.4280(0.953)$ & $2.1784(0.908)$ \\
\hline & & Boot-p & $1.5180(0.986)$ & $1.3912(0.980)$ & $1.5361(0.953)$ \\
\hline & & Boot-t & $1.4893(0.977)$ & $1.2220(0.972)$ & $1.8852(0.948)$ \\
\hline & & MCMC & $1.0195(0.995)$ & $1.0224(0.989)$ & $2.0424(0.980)$ \\
\hline \multirow{12}{*}{$(50,25)$} & \multirow[t]{4}{*}{$\left(25,0^{* 24}\right)$} & AMLE & $1.6822(0.948)$ & $2.0043(0.948)$ & $1.7254(0.918)$ \\
\hline & & Boot-p & $1.1591(0.988)$ & $1.2223(0.976)$ & $1.3896(0.959)$ \\
\hline & & Boot-t & $1.1233(0.980)$ & $1.0825(0.963)$ & $1.6813(0.956)$ \\
\hline & & MCMC & $0.7045(0.995)$ & $0.8015(0.995)$ & $1.1939(0.995)$ \\
\hline & \multirow[t]{4}{*}{$\left(0^{* 24}, 25\right)$} & AMLE & $2.4964(0.929)$ & $2.0865(0.943)$ & $1.8982(0.881)$ \\
\hline & & Boot-p & $1.6240(0.991)$ & $1.3451(0.969)$ & $1.4991(0.949)$ \\
\hline & & Boot-t & $1.4754(0.984)$ & $1.3215(0.965)$ & $1.9777(0.950)$ \\
\hline & & MCMC & $1.0548(0.990)$ & $0.8530(0.992)$ & $1.6332(0.989)$ \\
\hline & \multirow[t]{4}{*}{$\left(1^{* 25}\right)$} & AMLE & $2.0768(0.952)$ & $2.0207(0.951)$ & $1.7735(0.893)$ \\
\hline & & Boot-p & $1.4209(0.987)$ & $1.2578(0.978)$ & $1.4285(0.955)$ \\
\hline & & Boot-t & $1.3983(0.981)$ & $1.1819(0.969)$ & $1.7906(0.951)$ \\
\hline & & MCMC & $0.8841(0.994)$ & $0.8287(0.995)$ & $1.3578(0.985)$ \\
\hline
\end{tabular}


Table 8: (Continued)

\begin{tabular}{|c|c|c|c|c|c|}
\hline$(n, m)$ & Scheme & Methods & $b$ & $a$ & $k$ \\
\hline \multirow{8}{*}{$(50,35)$} & \multirow{8}{*}{$\left(0^{* 34}, 15\right)$} & AMLE & $1.5312(0.935)$ & $1.8924(0.942)$ & $1.6558(0.923)$ \\
\hline & & Boot-p & $1.0502(0.982)$ & $1.1449(0.968)$ & $1.3466(0.960)$ \\
\hline & & Boot-t & $1.0091(0.976)$ & $1.0229(0.960)$ & $1.5397(0.956)$ \\
\hline & & MCMC & $0.6321(0.995)$ & $0.7296(0.996)$ & $1.0488(0.992)$ \\
\hline & & AMLE & $2.0751(0.938)$ & $2.0139(0.954)$ & $1.7742(0.904)$ \\
\hline & & Boot-p & $1.2778(0.989)$ & $1.1525(0.975)$ & $1.4394(0.953)$ \\
\hline & & Boot-t & $1.1310(0.982)$ & $1.1002(0.966)$ & $1.8350(0.950)$ \\
\hline & & MCMC & $0.7835(0.989)$ & $0.7491(0.991)$ & $1.2606(0.987)$ \\
\hline \multirow{16}{*}{$(50,45)$} & \multirow{4}{*}{$\left(0^{* 10}, 1^{* 15}, 0^{* 10}\right)$} & AMLE & $1.4405(0.942)$ & $1.9162(0.947)$ & $1.6804(0.913)$ \\
\hline & & Boot-p & $1.1314(0.984)$ & $1.1488(0.973)$ & $1.3243(0.952)$ \\
\hline & & Boot-t & $1.1243(0.978)$ & $1.0785(0.964)$ & $1.6680(0.952)$ \\
\hline & & MCMC & $0.6846(0.992)$ & $0.7375(0.995)$ & $1.0881(0.990)$ \\
\hline & \multirow[t]{4}{*}{$\left(5,0^{* 44}\right)$} & AMLE & $1.4793(0.929)$ & $1.8323(0.939)$ & $1.5814(0.908)$ \\
\hline & & Boot-p & $0.9856(0.987)$ & $1.0974(0.971)$ & $1.2944(0.954)$ \\
\hline & & Boot-t & $0.9875(0.980)$ & $1.0214(0.962)$ & $1.4041(0.948)$ \\
\hline & & MCMC & $0.5694(0.993)$ & $0.6735(0.993)$ & $0.9212(0.993)$ \\
\hline & \multirow[t]{4}{*}{$\left(0^{* 44}, 5\right)$} & AMLE & $1.5716(0.936)$ & $1.9491(0.938)$ & $1.6373(0.897)$ \\
\hline & & Boot-p & $1.0545(0.981)$ & $1.0961(0.973)$ & $1.3715(0.956)$ \\
\hline & & Boot-t & $1.0543(0.976)$ & $1.0814(0.965)$ & $1.6192(0.953)$ \\
\hline & & MCMC & $0.6354(0.983)$ & $0.6864(0.990)$ & $0.9602(0.986)$ \\
\hline & \multirow[t]{4}{*}{$\left(0^{* 20}, 1^{* 5}, 0^{* 20}\right)$} & AMLE & $1.3767(0.927)$ & $1.9116(0.931)$ & $1.6145(0.915)$ \\
\hline & & Boot-p & $1.0119(0.982)$ & $1.0957(0.971)$ & $1.2836(0.951)$ \\
\hline & & Boot-t & $1.0104(0.975)$ & $1.0336(0.964)$ & $1.5208(0.949)$ \\
\hline & & MCMC & $0.5721(0.987)$ & $0.6882(0.985)$ & $0.9504(0.995)$ \\
\hline
\end{tabular}

From Tables 3-6, it is observed that the ERs of all estimates decrease as the sample sizes increase in all cases, as expected. The results shows that the MLEs using the EM algorithm have smaller ERs than the MLEs using the NR algorithm. The performances of the MLEs using the EM algorithm are satisfactory, especially when the sample size is small. For large sample sizes, we observe that the ERs of the MLEs using the EM algorithm and the Bayes estimators become very close in most of the cases. Also, it is clear that the Bayes estimates under LINEX loss function are sensitive to the values of the shape parameter $c$.

From Tables 7 and 8, we observe that the expected lengths of the intervals decrease as the sample sizes increase, as expected. The results shows that the performance of the approximate confidence intervals in comparison with bootstrap confidence intervals are not satisfactory, especially for small sample sizes. Comparing the bootstrap confidence intervals, we observe that the performance of Boot-p confidence intervals and Boot- $t$ confidence intervals are almost similar in terms of coverage probabilities but in terms of expected lengths the Boot-p confidence intervals provides the shorter expected lengths. In most of the cases, it is observed that the HPD credible intervals have the better performances in comparison with the other intervals, especially when the sample size is large.

In most of the cases, it is observed that the ERs of the estimators and the expected lengths of the intervals are maximized when the censoring is at the end of the experiment and are minimized when the censoring is at the first of the experiment. This may be occurred because, the data obtained when the censoring is at the first of the experiment would be expected to provide more information about the unknown parameters than the data obtained when the censoring is at the end of the experiment. 


\section{Conclusions}

In this paper, we consider a new class of hazard rate distributions called the powerlinear hazard rate distribution. This distribution is obtained by combining the linear hazard rate function and the power hazard rate function and it contains a number of known lifetime distributions such as linear hazard rate distribution, power hazard rate distribution, exponential distribution, Rayleigh distribution and Weibull distribution. The power-linear hazard rate function can cover constant, decreasing, increasing and bathtub-shaped hazard rates. The MLEs via the EM and NR algorithms and Bayesian estimation of the parameters of the power-linear hazard rate distribution under SEL, LINEX and Stein loss functions are obtained. The asymptotic confidence interval and the HPD credible interval of the parameters are also discussed. A real data set is analyzed in order to illustrate the inferences discussed in the previous sections. It observed that the present distribution can provide a better fit than its sub-models and some threeparameter distributions. Finally, to investigate and compare the performance of different types of estimators presented in this paper, a Monte Carlo simulation study is conducted. Based on simulation results, we observed that the MLEs using the EM algorithm work well, especially when the sample size is small. Also, the performance of the Bayes estimators and the HPD credible intervals based on the MCMC method are very good, especially for large sample sizes.

\section{Appendix}

Using (3.13), the required conditional expectations in the EM algorithm are obtained in the following form

$$
E\left(Z_{j l}^{2} \mid x_{j: m: n}, b, a, k\right)=e^{\frac{b}{2} x_{j: m: n}^{2}+\frac{a}{k+1} x_{j: m: n}^{k+1}} \int_{x_{j: m: n}}^{\infty}\left(b z_{j l}^{3}+a z_{j l}^{k+2}\right) e^{-\frac{b}{2} z_{j l}^{2}-\frac{a}{k+1} z_{j l}^{k+1}} d z_{j l} .
$$

Using the following Taylor expansion

$$
e^{-\frac{a}{k+1} z^{k+1}}=\sum_{i=0}^{\infty} \frac{(-1)^{i}}{i !}\left(\frac{a}{k+1}\right)^{i} z^{(k+1) i}
$$

and then by making the change of variable $v=b z^{2} / 2$, we have

$$
\begin{aligned}
& E\left(Z_{j l}^{2} \mid x_{j: m: n}, b, a, k\right)=e^{\frac{b}{2} x_{j: m: n}^{2}+\frac{a}{k+1} x_{j: m: n}^{k+1}} \sum_{i=0}^{\infty} \frac{(-1)^{i}}{i !}\left(\frac{a}{k+1}\right)^{i} \\
& \times\left\{\left(\frac{2}{b}\right)^{\frac{i}{2}(k+1)+1} \Gamma\left(\frac{i}{2}(k+1)+2, \frac{b}{2} x_{j: m: n}^{2}\right)\right. \\
& \left.+\frac{a}{b}\left(\frac{2}{b}\right)^{\frac{1}{2}(i+1)(k+1)} \Gamma\left(\frac{1}{2}(i+1)(k+1)+1, \frac{b}{2} x_{j: m: n}^{2}\right)\right\},
\end{aligned}
$$

where $\Gamma(a, b)=\int_{b}^{\infty} x^{a-1} e^{-x} d x$ is the upper incomplete gamma function. Similarly,

$$
\begin{aligned}
& E\left(Z_{j l}^{k+1} \mid x_{j: m: n}, b, a, k\right)=e^{\frac{b}{2} x_{j: m: n}^{2}+\frac{a}{k+1} x_{j: m: n}^{k+1}} \sum_{i=0}^{\infty} \frac{(-1)^{i}}{i !}\left(\frac{a}{k+1}\right)^{i} \\
& \times\left\{\left(\frac{2}{b}\right)^{\frac{1}{2}(i+1)(k+1)} \Gamma\left(\frac{1}{2}(i+1)(k+1)+1, \frac{b}{2} x_{j: m: n}^{2}\right)\right. \\
& \left.+\frac{a}{b}\left(\frac{2}{b}\right)^{\frac{i}{2}(k+1)+k} \Gamma\left(\frac{i}{2}(k+1)+k+1, \frac{b}{2} x_{j: m: n}^{2}\right)\right\} \\
& E\left(\frac{Z_{j l}}{b Z_{j l}+a Z_{j l}^{k}} \mid x_{j: m: n}, b, a, k\right)=e^{\frac{b}{2} x_{j: m: n}^{2}+\frac{a}{k+1} x_{j: m: n}^{k+1}} \sum_{i=0}^{\infty} \frac{(-1)^{i}}{i !}\left(\frac{a}{k+1}\right)^{i}
\end{aligned}
$$




$$
\begin{aligned}
& \times \frac{1}{b}\left(\frac{2}{b}\right)^{\frac{i}{2}(k+1)} \quad \Gamma\left(\frac{i}{2}(k+1)+1, \frac{b}{2} x_{j: m: n}^{2}\right), \\
& E\left(\frac{Z_{j l}^{k}}{b Z_{j l}+a Z_{j l}^{k}} \mid x_{j: m: n}, b, a, k\right)=e^{\frac{b}{2} x_{j: m: n}^{2}+\frac{a}{k+1} x_{j: m: n}^{k+1}} \sum_{i=0}^{\infty} \frac{(-1)^{i}}{i !}\left(\frac{a}{k+1}\right)^{i} \\
& \times \frac{1}{b}\left(\frac{2}{b}\right)^{\frac{1}{2}(i(k+1)+k-1)} \quad \Gamma\left(\frac{1}{2}(i+1)(k+1), \frac{b}{2} x_{j: m: n}^{2}\right), \\
& E\left(\frac{Z_{j l}^{k} \ln Z_{j l}}{b Z_{j l}+a Z_{j l}^{k}} \mid x_{j: m: n}, b, a, k\right)=e^{\frac{b}{2} x_{j: m: n}^{2}+\frac{a}{k+1} x_{j: m: n}^{k+1}} \sum_{i=0}^{\infty} \frac{(-1)^{i}}{i !}\left(\frac{a}{k+1}\right)^{i} \\
& \times \frac{1}{2 b}\left(\frac{2}{b}\right)^{\frac{1}{2}(i(k+1)+k-1)} \quad \int_{\frac{b}{2} x_{j: m: n}^{2}}^{\infty} \ln \left(\frac{2 v}{b}\right) v^{\frac{1}{2}(i(k+1)+k-1)} e^{-v} d v, \\
& E\left(Z_{j l}^{k+1} \ln Z_{j l} \mid x_{j: m: n}, b, a, k\right)=e^{\frac{b}{2} x_{j: m: n}^{2}+\frac{a}{k+1} x_{j: m: n}^{k+1}} \sum_{i=0}^{\infty} \frac{(-1)^{i}}{i !}\left(\frac{a}{k+1}\right)^{i} \\
& \times\left\{\frac{1}{2}\left(\frac{2}{b}\right)^{\frac{1}{2}(i+1)(k+1)} \int_{\frac{b}{2} x_{j: m: n}^{2}}^{\infty} \ln \left(\frac{2 v}{b}\right) v^{\frac{1}{2}(i+1)(k+1)} e^{-v} d v\right. \\
& \left.+\frac{a}{2 b}\left(\frac{2}{b}\right)^{\frac{i}{2}(k+1)+k} \int_{\frac{b}{2} x_{j: m: n}^{2}}^{\infty} \ln \left(\frac{2 v}{b}\right) v^{\frac{i}{2}(k+1)+k} e^{-v} d v\right\} .
\end{aligned}
$$

Note that the right hand side of (7.2)-(7.7) does not depend on $l$, so, we can eliminate the index $l$ from $z_{j l}$.

\section{References}

[1] Akdam, N., Kinaci, I. and Saracoglu, B. Statistical inference of stress-strength reliability for the exponential power distribution based on progressive type-II censored samples, Hacettepe Journal of Mathematics and Statistics 46, 239-253, 2017.

[2] Bain, L. Analysis for the linear failure-rate life testing distribution, Technometrics 15, 551559, 1974.

[3] Balakrishnan, N. and Aggarwala, R. Progressive Censoring, Theory, Methods and Applications, Birkhauser, Boston, 2000.

[4] Balakrishnan, N. and Sandhu, R.A. A simple simulation algorithm for generating progressively type-II generated samples, American Statistician 49, 229-230, 1995.

[5] Chen, M.H. and Shao, Q.M. Monte Carlo estimation of Bayesian credible and HPD intervals, Journal of Computational and Graphical Statistics 8, 69-92, 1999.

[6] Cohen, A.C. Progressively censored samples in life testing, Technometrics 5, 327-339, 1963.

[7] Cordeiro G.M., Ortega, E.M. and Popovic, B.V. The gamma-linear failure rate distribution: theory and applications, Journal of Statistical Computation and Simulation 84, 2408-2426, 2014.

[8] Dempster, A.P., Laird, N.M. and Rubin, D.B. Maximum likelihood from incomplete data via the EM algorithm, J. Roy. Statist. Soc. Ser. B 39, 1-38, 1977.

[9] Efron, B. and Tibshirani, R.J. An Introduction to the Bootstrap, Chapman and Hall, New York, 1993.

[10] Gelfand, A.E. and Smith, A.F.M. Sampling-based approaches to calculating marginal densities, Journal of the American Statistical Association 85, 398-409, 1990.

[11] Ghitany, M.E. and Kotz, S. Reliability properties of extended linear failure rate distributions, Probability in the Engineering and Informational Sciences 21, 441-450, 2007.

[12] Jiang, H., Xie, M. and Tang, L.C. On MLEs of the parameters of a modified Weibull distribution for progressively type-II censored samples, Journal of Applied Statistics 37, 617-627, 2010.

[13] Kinaci, I. Estimation of $P(Y<X)$ for distributions having power hazard function, Pakistan Journal of Statistics 30, 57-70, 2014. 
[14] Kus, C. and Kaya, M.F. Estimation of parameters of the loglogistic distribution based on progressive censoring using the EM algorithm, Hacettepe Journal of Mathematics and Statistics 35, 203-211, 2006.

[15] Lin, C.T., Wu. S.J.S. and Balakrishnan, N. Parameter estimation for the linear hazard rate distribution based on records and inter-record times, Communications in Statistics-Theory and Methods 32, 729-748, 2003.

[16] Lin, C.T., Wu. S.J.S. and Balakrishnan, N. Monte Carlo methods for Bayesian inference on the linear hazard rate distribution, Communications in Statistics-Simulation and Computation 35, 575-590, 2006.

[17] Linhart, H. and Zucchini, W. Model Selection, Wiley, New York, 1986.

[18] Makeham, W.M. On the law of mortality and the construction of annuity tables, J. Inst. Actuar. Assur. Mag. 8, 301-310, 1860.

[19] Miller, R.G. Survival Analysis, John Wiley, New York, 1981.

[20] Mugdadi, A.R. The least squares type estimation of the parameters in the power hazard function, Applied Mathematics and Computation 169, 737-748, 2005.

[21] Mugdadi, A.R. and Min, A. Bayes estimation of the power hazard function, Journal of Interdisciplinary Mathematics 12, 675-689, 2009.

[22] Ng, T., Chan, C.S. and Balakrishnan, N. Estimation of parameters from progressively censored data using EM algorithm, Computational Statistics and Data Analysis 39, 371-386, 2002.

[23] Pandey, A., Singh, A. and Zimmer, W.J. Bayes estimation of the linear hazard rate model, IEEE Transactions on Reliability 42, 636-640, 1993.

[24] Parsian, A. and Nematollahi, N. Estimation of scale parameter under entropy loss function, Journal of Statistical Planning and Inference 52, 377-391, 1996.

[25] Rastogi, M.K., Tripathi, Y.M. and Wu, S.J. Estimating the parameters of a bathtub-shaped distribution under progressive type-II censoring, Journal of Applied Statistics 39, 2389-2411, 2012.

[26] Rastogi, M.K. and Tripathi, Y.M. Parameter and reliability estimation for an exponentiated half-logistic distribution under progressive type-II censoring, Journal of Statistical Computation and Simulation 84, 1711-1727, 2014.

[27] Rinne, H. The hazard rate: theory and inference, Justus-Liebig University Press, 2014.

[28] Sarhan, A.M. and Kundu, D. Generalized linear failure rate distribution, Communications in statistics: Theory and Methods 38, 642-666, 2009.

[29] Sarhan, A.M. and Zaindin, M. Modified Weibull distribution, Applied Sciences 11, 123-136, 2009.

[30] Sen, A. and Bhattacharyya, G. Inference procedures for the linear failure rate model, Journal of Statistical Planning and Inference 46, 59-76, 1995.

[31] Sen, A., Kannan, N. and Kundu, D. Bayesian planning and inference of a progressively censored sample from linear hazard rate distribution, Computational Statistics and Data Analysis 63, 108-121, 2013

[32] Tian, Y., Tian, M. and ZHU, Q. A new generalized linear exponential distribution and its applications, Acta Mathematicae Applicatae Sinica, English Series 30, 1049-1062, 2014.

[33] Tierney, L. Markov chains for exploring posterior distributions, The Annals of Statistics 22, 1701-1728, 1994

[34] Varian, H. A Bayesian approach to real estate assessment, Studies in Bayesian Econometrics and Statistics in Honor of Leonard J. Savege, Amsterdam: North Holland 195-208, 1975. 